\title{
Fault-Tolerant Aircraft Control Based on Self- Constructing Fuzzy Neural Networks and Multivariable SMC under Actuator Faults
}

\author{
Xiang Yu, Senior Member, IEEE, Yu Fu, Peng Li, and Youmin Zhang, Senior Member, IEEE
}

\begin{abstract}
This paper presents a fault-tolerant aircraft control (FTAC) scheme against actuator faults. Firstly, the upper bounds of the norms of the unknown functions are introduced, which contain actuator faults and model uncertainties. Subsequently, self-constructing fuzzy neural networks (SCFNNs) with adaptive laws are capable of obtaining the bounds. The bound estimation can reduce the computational burden with a lower amount of rules and weights, rather than the dynamic matrix approximation. Moreover, with the aid of SCFNNs, a multivariable sliding mode control (SMC) is developed to guarantee the finite-time stability of the handicapped aircraft. As compared to the existing intelligent FTAC techniques, the proposed method has twofold merits: fault accommodation can be promptly accomplished and decoupled difficulties can be overcome. Finally, simulation results from the nonlinear longitudinal Boeing 747 aircraft model illustrate the capability of the presented FTAC scheme.
\end{abstract}

Index Terms-Fault-tolerant aircraft control; actuator faults; self-constructing fuzzy neural network; finite-time stability; multivariable sliding mode control.

\section{INTRODUCTION}

$\mathrm{W}$ ITH a high degree of integrating automation technologies, aerospace engineering systems have become increasingly vulnerable to anomalies caused by structure impairments, actuator/sensor faults, or other subsystem malfunctions. Each of the in-flight failures can alter aircraft characteristics, further undermining safety. Without any appropriate reactions engaged in a timely fashion, even a relatively minor error may develop into catastrophes.

Fault-tolerant aircraft control (FTAC) designs to maintain flight safety can be essentially classified into passive and active approaches [1-3]. Within a passive FTAC context, one flight controller is usually developed with consideration for both normal and faulty cases. The resulting control thereby makes the closed-loop system invulnerable to the anticipated faults without any control structure or parameter adjustment. This

This work was supported in part by Natural Sciences and Engineering Research Council of Canada, in part by the National Natural Science Foundation of China under Grant 61403407, Grant 61573282, and Grant 61603130 , and in part by SPNSF under Grant 2015JZ020.

Xiang Yu is with the Department of Mechanical, Industrial and Aerospace Engineering, Concordia University, Montreal, Quebec, H3G 1M8, Canada (Email: xiangyu1110@gmail.com).

$\mathrm{Yu} \mathrm{Fu}$ is with the State Grid Zhangzhou Electric Power Supply Company. She was with the Department of Mechanical, Industrial and Aerospace Engineering, Concordia University, Montreal, Quebec, H3G 1M8, Canada (Email: yufu1013@gmail.com).

Peng $\mathrm{Li}$ is with the College of Mechatronics Engineering and Automation, National University of Defense Technology, Changsha, 410073, China (Email: lipeng_2010@163.com).

Youmin Zhang (Corresponding Author) is with the Department of Mechanical, Industrial and Aerospace Engineering, Concordia University, Montreal, Quebec, H3G 1M8, Canada (E-mail: ymzhang @encs.concordia.ca). type of FTAC provides accommodation for faults from a "passive" viewpoint. On the other hand, the principle of active FTAC is to reconfigure the flight controller in response to the knowledge of the current state of the aircraft. Thus, the term "active" implies that corrective actions are triggered to handle the identified system/component malfunctions.

The past decades have witnessed the development of various FTAC technologies. 1) With respect to passive FTAC, the eigenvalue assignment technique [4] and multi-objective optimization approach $[5,6]$ are exploited for preserving the asymptotic stability of the handicapped aircraft and an acceptable level of performance. However, feasible solutions may not be found if excessive quantities of fault scenarios are prescribed in the design phase of passive FTAC. 2) Active FTAC systems are developed based on a variety of control technologies. To mention a few, model predictive control (MPC) [7], backstepping control [8, 9], adaptive control [10-14], sliding mode control (SMC) [15-17], and linear parameter varying (LPV) control [18] techniques are exploited to reconfigure the control corresponding to in-flight faults. Within an active FTAC scheme, the accuracy of fault detection and diagnosis (FDD) and switching time of reconfigured control have a predominant impact on fault tolerant performance [2,3].

Additionally, by resorting to fuzzy techniques, several results in the literature are available not only to improve the safety of other engineering systems [19,20], but also to advance the state of the art of FTAC designs. In [21], a fuzzy model reference learning control technique is deployed to counteract the effects of aileron stuck failures. Moreover, an expert supervisory mechanism enables the flight safety without explicit FDD results. A sequential adaptive fuzzy inference system (SAFIS), which can update the rules, is adopted to approximate the aircraft dynamics [22]. As a consequence, the SAFIS-aided FTAC allows the aircraft to successfully land in spite of actuator failures. As reported in [23], fuzzy logic systems (FLSs) are employed to estimate amplitudes of actuator gain and bias faults. Then, the resulting adaptive controller attempts to guarantee the asymptotic stability of the near-space vehicle (NSV) subject to actuator malfunctions. The use of a TakagiSugeno (T-S) fuzzy model is established to describe the NSV dynamics [24-26]. An adaptive control approach is applied to alleviate adverse impacts of actuator faults [24, 26], while an adaptive observer is developed to identify sensor faults [25]. In [27], type-2 fuzzy logic control and SMC methods are combined to cope with aircraft actuator faults. The basic idea in [27] is to separate the FTAC into the pitch, roll, yaw, and altitude channels. More recently, the findings in [28, 29] show that the unknown nonlinear functions can be estimated by FLSs, while the asymptotic stability of the faulty aircraft can be maintained using SMC techniques. 
Although extensive design activities are conducted for aircraft safety, there are some difficulties that need to be addressed. 1) Model variations and FDD accuracy are well recognized as two important factors affecting FTAC performance $[2,3]$. Any real continuous functions on a compact set can be approximated to an arbitrary accuracy using a FLS. Hence, a FLS has potential for estimating aircraft dynamics and aircraft faults. The adaptation capability of FLS with only output weights being updated is limited due to the fact that the regressors are fixed [23, 28-31]. By taking advantage of neural networks (NNs) [32], self-constructing fuzzy systems are capable of updating fuzzy rules under system operating conditions [22, 33, 34]. Nevertheless, the computational cost is substantially increased as the quantity of rules and weights increases. As aforementioned, determining how to exploit a fuzzy system with a high level of adaptation capability and a low level of computation burden is very challenging for FTAC design. 2) Time available for fault recovery depends solely on fault nature and flight conditions [2, 3]. In flight, the time frame of faulty aircraft developing into an irreversible state is typically a few seconds. More specifically, actuator malfunctions can quickly drive the faulty aircraft out of control without prompt reactions exposed. Thus, for preventing aircraft breakup, fault accommodation must be accomplished in a timely manner. However, the existing FTAC based on fuzzy strategies can only guarantee the asymptotic stability of the handicapped aircraft [24, 26-29]. 3) In most of FTAC systems based on both fuzzy and SMC techniques, the design problem is often formulated as the decoupled problem with $m$ singleinput structures. By contrast, aircraft aerodynamics exhibits strong couplings. For instance, in addition to contributing to rolling maneuvers, ailerons can affect pitching and yawing motions. Thus, accounting for multivariable situations may be more appropriate for FTAC design rather than the decoupled treatment.

In an attempt to overcome the discussed difficulties, this paper presents new developments in the integration of selfconstructing fuzzy neural network (SCFNN) and multivariable SMC methods into a FTAC system against actuator faults. Since the proposed FTAC can actively counteract actuator faults, it can be seen as an active FTAC scheme. The major contributions are briefly outlined by three aspects.

1) SCFNNs, which can be continuously running to update both the structures and parameters, are incorporated into adaptive techniques. Consequently, the upper bounds of the norms of unknown functions including actuator fault amplitudes and model uncertainties can be captured. When comparing to the previous studies [23, 27-29], the proposed algorithm with the learning property in response to actuator faults can achieve superior approximation performance and facilitate fault accommodation. Furthermore, estimating the bounds helps in reducing the computational burden with a lower amount of rules and weights, as opposed to approximating the overall dynamics [22, 33, 34].

2) A SMC approach is deployed in the proposed FTAC scheme. The trajectory of the faulty system can be steered to the equilibrium within finite time as long as the sliding surface is reached. Thus, the resulting FTAC can ensure the finite-time stability of the aircraft, even under conditions involving actuator faults and model uncertainties. This feature sets this study apart from the similar works [24, 2629], based on which the stability of post-fault aircraft is asymptotically guaranteed. Hence, the integration of finitetime SMC allows the developed scheme to improve flight safety.

3) The FTAC based on SCFNNs and SMC is designed for multivariable situations. The so-called multivariable SMC is formed by vector expression, which is successfully incorporated in the FTAC design. In contrast to [27], the decoupled issue can be avoided in the proposed algorithm by incorporating the multivariable SMC approach. Therefore, the proposed design becomes more proper for the cases where strong couplings are inherent to aircraft aerodynamics.

The remainder of this paper is arranged as follows. Aircraft longitudinal model and actuator fault model are presented in Section II. The principle of SCFNN is described in Section III. A FTAC scheme is developed to counteract actuator failures within finite time in Section IV, where the SCFNNs and the multivariable SMC are integrated. In Section $\mathrm{V}$, the performance of the proposed FTAC scheme is evaluated through simulation studies based on a longitudinal model of Boeing 747 aircraft. Section VI includes a discussion of the conclusions.

\begin{tabular}{ll}
\multicolumn{1}{c}{ TABLE I } \\
NOMENCLATURE
\end{tabular}

\section{AirCRAFt Longitudinal Model}

\section{A. Aircraft Dynamics}

Even though the analysis and the design approaches are not limited to a specific type of aircraft in this work, it is advantageous to work with a specific aircraft system to explain the concepts and to validate the design procedure. The Boeing 747 series 100/200, as one of the most popular and widely used wide-body commercial jet airliners, is used as an example in 
this research to illustrate the FTAC design procedure. According to [35], the body-axes longitudinal motion of the Boeing 747 without considering flexible effects can be represented as:

$$
\begin{gathered}
\dot{q}=c_{7} M_{y}, \\
\dot{V}=\frac{F_{x} \cos \alpha+F_{z} \sin \alpha}{m} .
\end{gathered}
$$

The body-axis aerodynamic forces and moments are described as:

$$
\begin{aligned}
F_{x}= & -\bar{q} S_{r}\left(C_{D} \cos \alpha-C_{L} \sin \alpha\right)+T-m g \sin \theta \\
F_{z}= & -\bar{q} S_{r}\left(C_{D} \sin \alpha+C_{L} \cos \alpha\right)-0.0436 T+m g \cos \theta \\
M_{y}= & \bar{q} S_{r} \bar{c}\left[C_{m}-\frac{1}{\bar{c}}\left(C_{D} \sin \alpha+C_{L} \cos \alpha\right) \bar{x}_{c g}\right. \\
- & \left.\frac{1}{\bar{c}}\left(C_{D} \cos \alpha-C_{L} \sin \alpha\right) \bar{z}_{c g}+\frac{\bar{c} \dot{\alpha}}{V}\left(C_{m_{\dot{\alpha}}}-\frac{\bar{x}_{c g}}{\bar{c}} C_{L_{\dot{\alpha}}} \cos \alpha\right)\right] \\
& +z_{\text {eng }} T .
\end{aligned}
$$

The aerodynamic coefficients for the longitudinal motion can be expressed as:

$$
\begin{gathered}
C_{L}=C_{L 0}+\frac{\bar{c}}{2 V}\left(1.45-1.8 \bar{x}_{c g}\right) \frac{d C_{L}}{d q} q \\
+K_{\alpha}\left(\frac{d C_{L}}{d \delta_{i e}} \delta_{i e}+\frac{d C_{L}}{d \delta_{o e}} \delta_{o e}\right) \\
C_{D}=C_{D_{M a c h}}, \\
C_{m}=C_{m 0}+\frac{\bar{c}}{2 V} \frac{d C_{m}}{d q} q+K_{\alpha} \frac{d c_{m}}{d \delta_{s}} \delta_{s}+K_{\alpha}\left(\frac{d C_{m}}{d \delta_{i e}} \delta_{i e}+\frac{d C_{m}}{d \delta_{o e}} \delta_{o e}\right) .
\end{gathered}
$$

Furthermore, the aerodynamic coefficients can be approximated as polynomial functions of AOA and velocity over the flight regime [36]:

$$
\begin{gathered}
C_{D_{M a c h}}=\kappa_{20} \alpha^{2}+\kappa_{10} \alpha+\kappa_{01} V+\kappa_{00}, \\
\frac{d C_{L}}{d \delta_{i e}}=\frac{d C_{L}}{d \delta_{o e}}=\tau_{02} V^{2}+\tau_{01} V+\tau_{00}, \\
C_{L 0}=\eta_{10} \alpha+\eta_{01} V+\eta_{00}, \\
C_{m 0}=\xi_{20} \alpha^{2}+\xi_{10} \alpha+\xi_{01} V+\xi_{00}, \\
\frac{d C_{m}}{d \delta_{i e}}=\frac{d C_{m}}{d \delta_{o e}}=\zeta_{02} V^{2}+\zeta_{01} V+\zeta_{00},
\end{gathered}
$$

where

$$
\left\{\begin{array}{l}
\kappa_{20}=3.27, \kappa_{10}=3.48 \times 10^{-2}, \kappa_{01}=4.45 \times 10^{-5}, \kappa_{00}=9.92 \times 10^{-3} \\
\tau_{02}=-0.72 \times 10^{-7}, \tau_{01}=2.13 \times 10^{-5}, \tau_{00}=1.61 \times 10^{-3} \\
\eta_{10}=5.15, \eta_{01}=1.21 \times 10^{-3}, \eta_{00}=6.15 \times 10^{-3} \\
\xi_{20}=2.39, \xi_{10}=-1.46, \xi_{01}=-3.20 \times 10^{-4}, \xi_{00}=0.12 \\
\zeta_{02}=2.18 \times 10^{-7}, \zeta_{01}=-0.58 \times 10^{-4}, \zeta_{00}=-0.88 \times 10^{-2}
\end{array}\right.
$$

Remark 1: From Eq. (6), it is known that the lift coefficient $C_{L}$ is based on the effects of the pitch angular rate, the elevator deflections, and the basic component $C_{L 0}$, respectively. Eq. (7) indicates that the drag coefficient $C_{D}$ greatly relies on the effect of the Mach number. As can be observed from Eq. (8), the essential factors affecting the pitch moment coefficient $C_{m}$ contain the pitch angular rate, the inner elevator deflection, the outer elevator deflection, the stabilizer deflection, and the basic component $C_{m 0}$, respectively.

Substituting Eqs. (3)-(8) into Eqs. (1)-(2) gives:

$$
\begin{aligned}
\dot{q}= & \frac{c_{7} \bar{q} S_{r} \bar{c}}{2 V}\left[\bar{c} \frac{d C_{m}}{d q}-\left(1.45-1.8 \bar{x}_{c g}\right)\left(\bar{x}_{c g} \cos \alpha+\bar{z}_{c g} \sin \alpha\right)\right] q \\
& +c_{7} \bar{q} S_{r} \bar{c} C_{m 0}+c_{7} \bar{q} S_{r}\left[C_{D_{M a c h}}\left(\bar{z}_{c g} \cos \alpha-\bar{x}_{c g} \sin \alpha\right)\right. \\
& \left.-C_{L 0}\left(\bar{x}_{c g} \cos \alpha+\bar{z}_{c g} \sin \alpha\right)\right] \\
& +c_{7} \bar{q} S_{r} K_{\alpha}\left[\bar{c} \frac{d c_{m}}{d \delta_{i e}}-\left(\bar{x}_{c g} \cos \alpha+\bar{z}_{c g} \sin \alpha\right) \bar{c} \frac{d c_{L}}{d \delta_{i e}}\right] \delta_{i e} \\
& +c_{7} \bar{q} S_{r} K_{\alpha}\left[\bar{c} \frac{d c_{m}}{d \delta_{o e}}-\left(\bar{x}_{c g} \cos \alpha+\bar{z}_{c g} \sin \alpha\right) \bar{c} \frac{d C_{L}}{d \delta_{o e}}\right] \delta_{o e} \\
& +c_{7} \bar{q} S_{r} K_{\alpha} \bar{c} \frac{d C_{m}}{d \delta_{s}} \delta_{s}+c_{7} Z_{e n g} T \\
& \dot{V}=-g \sin \gamma-\frac{\bar{q} S_{r}}{m} C_{D_{\text {Mach }}}+\frac{4(\cos \alpha-0.0436 \sin \alpha)}{m} T .
\end{aligned}
$$

The aircraft parameters cannot be obtained precisely, leading to the challenges for flight control design. In common practice, there exist additive parameter perturbations $\left(\Delta_{*}\right)$ to the nominal values:

$$
\left\{\begin{array}{l}
m=m_{0}\left(1+\Delta_{m}\right) \\
S_{r}=S_{r 0}\left(1+\Delta_{S_{r}}\right) \\
I_{y y}=I_{y y 0}\left(1+\Delta_{I_{y y}}\right) . \\
\bar{c}=\bar{c}_{0}\left(1+\Delta_{\bar{c}}\right) \\
\bar{q}=\bar{q}_{0}\left(1+\Delta_{\bar{q}}\right)
\end{array}\right.
$$

By defining $\boldsymbol{x}=[q, V]^{T}$ and $\boldsymbol{u}=\left[\delta_{i e}, \delta_{o e}, \delta_{s}\right]^{T}$, the longitudinal motion equations can be simplified as:

$$
\begin{aligned}
\dot{\boldsymbol{x}} & =\boldsymbol{f}(\boldsymbol{x})+\boldsymbol{g}(\boldsymbol{x}) \boldsymbol{u} \\
& =\left(\boldsymbol{f}_{0}(\boldsymbol{x})+\Delta_{f}\right)+\left(g_{0}(\boldsymbol{x})+\Delta_{g}\right) \boldsymbol{u},
\end{aligned}
$$

where $\boldsymbol{f}(\boldsymbol{x}) \in \mathcal{R}^{2}$ and $\boldsymbol{g}(\boldsymbol{x}) \in \mathcal{R}^{2 \times 3}$ are smooth nonlinear functions of $\boldsymbol{x} . \boldsymbol{f}_{0}(\boldsymbol{x})$ and $\boldsymbol{g}_{0}(\boldsymbol{x})$ stand for the nominal terms of $\boldsymbol{f}(\boldsymbol{x})$ and $\boldsymbol{g}(\boldsymbol{x})$, while $\boldsymbol{\Delta}_{f}$ and $\boldsymbol{\Delta}_{g}$ denote the uncertain terms (modeling errors/uncertainties) of $\boldsymbol{f}(\boldsymbol{x})$ and $\boldsymbol{g}(\boldsymbol{x})$, respectively.

Remark 2: Linearized models based on small perturbation theory are often used at the flight control design stage. Even though the control design is relatively simple using the linearized model, the performance may be greatly degraded when the resulting control is engaged in a realistic environment. Furthermore, LPV [18] and T-S fuzzy [22-26] modelling techniques are recently applied to approximate aircraft nonlinear dynamics. The basic idea is to linearize the aircraft model at specific operating points and establish the relationship between these points. Nonetheless, model approximation accuracy and computational burden are recognized as major challenges. In this study, the nonlinear model as Eq. (18) is established to describe the nonlinear aircraft characteristics. Due to the lack of modeling technologies and experimental data, aerodynamic coefficients and relevant parameters cannot be 
obtained precisely. By considering this fact, model uncertainty is included as well in Eq. (18).

\section{B. Actuator Fault Model}

Actuators that can generate appropriate forces and moments are key components in any aircraft. Desired maneuvers can be completed if actuation systems work under a normal condition. On the contrary, poor performance and even instability are induced by actuator malfunctions. Gain fault appearing on an actuator is thought as a multiplicative-type fault, which deteriorates actuator effectiveness. Actuator bias fault as an additive-type fault creates a specific drift from the true amplitude. Since both gain fault and bias fault are concerned in this study, the model of actuator faults is represented as:

$$
\boldsymbol{u}_{F}=\boldsymbol{\Lambda} \boldsymbol{u}+\boldsymbol{\tau},
$$

where $\Lambda=\operatorname{diag}\left\{\lambda_{1}, \lambda_{2}, \lambda_{3}\right\}$ is used to describe the gain fault and $\boldsymbol{\tau}=\left[\tau_{1}, \tau_{2}, \tau_{3}\right]^{T}$ denotes the bias fault, respectively. Note that $0<\lambda_{i} \leq 1$ for $i=1,2,3$.

Remark 3: It is reported that the leakage of hydraulic fluid can be the root cause of degrading the actuator effectiveness [37]. Therefore, $\boldsymbol{\lambda}=\operatorname{diag}\left\{\lambda_{1}, \lambda_{2}, \lambda_{3}\right\}$ in Eq. (19) can be seen as the indicators of actuators effectiveness, where $0<\lambda_{1}, \lambda_{2}, \lambda_{3} \leq$ 1. In addition, a flight actuation system consists of an actuator controller, an actuator, and embedded sensors. The sensor fault in an actuator system can attribute to actuator bias faults. If the amplitude sensor encounters a bias fault, the measured amplitude is the actual amplitude plus the bias value. The sensed amplitude is mandated to follow the referenced signal. However, the actual value of the actuator amplitude is deviated from the one as required by the flight control. Hence, $\boldsymbol{\tau}=$ $\left[\tau_{1}, \tau_{2}, \tau_{3}\right]^{T}$ in Eq. (19) can represent bias faults of the inner elevator, outer elevator, and stabilizer, respectively.

Therefore, the corresponding expression for the aircraft longitudinal motion can be further represented upon Eq. (18) by taking into consideration of the actuator faults as follows:

$$
\dot{\boldsymbol{x}}=\left(\boldsymbol{f}_{0}(\boldsymbol{x})+\boldsymbol{\Delta}_{f}\right)+\left(\boldsymbol{g}_{0}(\boldsymbol{x})+\boldsymbol{\Delta}_{g}\right)(\boldsymbol{\Lambda} \boldsymbol{u}+\boldsymbol{\tau}) .
$$

Assumption 1: It is assumed that the following inequalities hold:

$$
\begin{gathered}
\left\|\boldsymbol{\Delta}_{f}+\left(\boldsymbol{g}_{0}+\boldsymbol{\Delta}_{g}\right) \boldsymbol{\tau}\right\| \leq \rho_{1}, \\
\left\|\boldsymbol{g}_{0}(\boldsymbol{\Lambda}-\boldsymbol{I}) \boldsymbol{g}_{0}^{+}+\boldsymbol{\Delta}_{g} \boldsymbol{\Lambda} \boldsymbol{g}_{0}^{+}\right\| \leq \rho_{2}<1,
\end{gathered}
$$

where $\boldsymbol{g}_{0}^{+}$is the pseudo inverse of $\boldsymbol{g}_{0}, \rho_{1}$ and $\rho_{2}$ are unknown positive parameters, respectively.

Remark 4: The term $\boldsymbol{\Delta}_{f}+\left(\boldsymbol{g}_{0}+\boldsymbol{\Delta}_{g}\right) \boldsymbol{\tau}$ is pertinent to the model uncertainties and the bias faults of actuators. A close look at $\boldsymbol{g}_{0}(\boldsymbol{\Lambda}-\boldsymbol{I}) \boldsymbol{g}_{0}^{+}+\boldsymbol{\Delta}_{g} \boldsymbol{\Lambda} \boldsymbol{g}_{0}^{+}$reveals that this term is associated with the uncertainty of control input matrix and the gain faults of actuators. The condition, $\| \boldsymbol{g}_{0}(\boldsymbol{\Lambda}-\boldsymbol{I}) \boldsymbol{g}_{0}^{+}+$ $\boldsymbol{\Delta}_{g} \boldsymbol{\Lambda} \boldsymbol{g}_{0}^{+} \|<1$, implies that $\boldsymbol{g}_{0} \boldsymbol{u}$ dominates the function $\boldsymbol{g}_{0}(\boldsymbol{\Lambda}-\boldsymbol{I}) \boldsymbol{u}+\boldsymbol{\Delta}_{g} \boldsymbol{\Lambda} \boldsymbol{u}$. This condition, in turn, ensures that the configured actuation systems possess adequate authority to counteract the considered faults.

\section{Problem Statement}

Even though the aircraft encounters actuator faults and model uncertainties, a FTAC system based on SCFNNs and multivariable SMC with adaptation techniques is proposed in this paper such that: 1) aircraft states can track the reference signals; and 2) the stability of the closed-loop system can be guaranteed within finite time.

\section{Self-Constructing FuZZy NeUral Networks}

SCFNN possesses the learning ability of NNs to tune the shape of the fuzzy membership functions and the output weights. In this study, the purpose of SCFNNs is to capture online the upper bounds $\left(\rho_{1}\right.$ and $\left.\rho_{2}\right)$ of the norms of unknown terms. It should be emphasized that the estimation process of $\rho_{1}$ and $\rho_{2}$ is time varying.

\section{A. SCFNN Architecture}

The SCFNN, sketched in Fig. 1, is comprised of four layers. Layer 1 receives the input variables. The membership values are calculated in Layer 2 such that the degree to which an input value associates with a fuzzy set can be determined. Precondition matching is carried out in Layer 3. The preconditions of the fuzzy rules are specified by the links before Layer 3, while the consequences are described by the succeeding links. Layer 4 is regarded as the output layer.
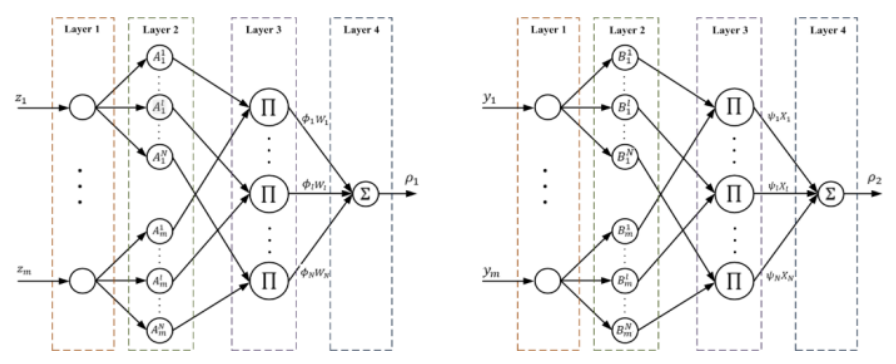

Fig. 1. Illustration of SCFNNs.

The rule base is:

$$
\mathrm{IF} z_{i} \text { is } A_{i}^{l}, \text { THEN } \rho_{1}(\mathbf{z})=W_{l},
$$

where $i=1,2, \cdots, m, l=1,2, \cdots, N, \rho_{1}: U_{z} \in \mathcal{R}^{m} \rightarrow \mathcal{R}, A_{i}^{l}$ denotes the membership value of the $i$ th input variable in the rule $l$, and $W_{l}$ is the output action strength related to the rule $l$.

The fuzzy basis function (FBF), $\phi_{l}$, is represented as:

$$
\phi_{l}=\exp \left\{-\frac{\left(\mathbf{z}-\boldsymbol{c}_{l}\right)^{T}\left(\mathbf{z}-\boldsymbol{c}_{l}\right)}{\Sigma_{l}^{2}}\right\}
$$

where $\boldsymbol{c}=\left[\boldsymbol{c}_{1}^{T}, \boldsymbol{c}_{2}^{T}, \cdots, \boldsymbol{c}_{N}^{T}\right]^{T} \in \mathcal{R}^{m N}, \boldsymbol{c}_{l}=\left[c_{l, 1}, c_{l, 2}, \cdots, c_{l, m}\right]^{T}$, $\boldsymbol{\sigma}=\left[\boldsymbol{\sigma}_{1}^{T}, \boldsymbol{\sigma}_{2}^{T}, \cdots, \boldsymbol{\sigma}_{N}^{T}\right]^{T} \in \mathcal{R}^{m N}, \boldsymbol{\sigma}_{l}=\left[\sigma_{l, 1}, \sigma_{l, 2}, \cdots, \sigma_{l, m}\right]^{T}, \boldsymbol{\Sigma}_{l}=$ $\operatorname{diag}\left\{\sigma_{l, 1}, \sigma_{l, 2}, \cdots, \sigma_{l, m}\right\} \in \mathcal{R}^{m \times m} \quad, \quad$ and $\quad l=1,2, \cdots, N$, respectively. $\boldsymbol{c}$ and $\boldsymbol{\sigma}$ denote the FBF center vector and the width vector, respectively.

For ease of notation, the output weight matrix $\boldsymbol{W}$ and the regressor vector $\boldsymbol{\phi}$ are specified as: 


$$
\begin{aligned}
& \boldsymbol{W}=\left[W_{1}, W_{2}, \cdots, W_{N}\right]^{T} \in \mathcal{R}^{N \times 1}, \\
& \boldsymbol{\phi}=\left[\phi_{1}, \phi_{2}, \cdots, \phi_{N}\right]^{T} \in \mathcal{R}^{N \times 1} .
\end{aligned}
$$

The SCFNN output with $N$ fuzzy rules are thereby described in a vector form:

$$
\rho_{1}(\mathbf{z})=\boldsymbol{W}^{T} \boldsymbol{\phi}(\mathbf{z} ; \boldsymbol{c}, \boldsymbol{\sigma})=\widehat{\boldsymbol{W}}^{T} \widehat{\boldsymbol{\phi}}(\mathbf{z} ; \widehat{\boldsymbol{c}}, \widehat{\boldsymbol{\sigma}})+\varepsilon_{1},
$$

where $\widehat{\boldsymbol{W}}$ and $\widehat{\boldsymbol{\phi}}$ are the estimates of $\boldsymbol{W}$ and $\boldsymbol{\phi}$, and $\varepsilon_{1}$ stands for the approximation error.

By adopting the SCFNN, $\rho_{2}$ can be approximated in a manner nearly identical to that described for $\rho_{1}$ :

$$
\rho_{2}(\boldsymbol{y})=\boldsymbol{X}^{T} \boldsymbol{\psi}(\boldsymbol{y} ; \boldsymbol{p}, \boldsymbol{q})=\widehat{\boldsymbol{X}}^{T} \widehat{\boldsymbol{\psi}}(\boldsymbol{y} ; \widehat{\boldsymbol{p}}, \widehat{\boldsymbol{q}})+\varepsilon_{2},
$$

where $\quad \boldsymbol{X}=\left[X_{1}, X_{2}, \cdots, X_{N}\right]^{T} \in \mathcal{R}^{N \times 1} \quad$ and $\quad \boldsymbol{\psi}=$ $\left[\psi_{1}, \psi_{2}, \cdots, \psi_{N}\right]^{T} \in \mathcal{R}^{N \times 1}$ denote the output weighting matrix and regressor vector, $\varepsilon_{2}$ specifies the approximation error, $\boldsymbol{p}$ and $\boldsymbol{q}$ represent the FBF center vector and the width vector, respectively.

Assumption 2: With respect to the SCFNN of $\rho_{1}$, all parameters are bounded on $U_{z}$, and

$$
\left\{\begin{array}{l}
\left|W_{l}\right| \leq \bar{W} \\
\underline{c}_{i} \leq\left|c_{l, i}\right| \leq \bar{c}_{i} \\
0<\underline{\sigma}_{i} \leq\left|\sigma_{l, i}\right| \leq \bar{\sigma}_{i}
\end{array} .\right.
$$

Focusing on the SCFNN of $\rho_{2}$, all the parameters are bounded on $U_{y}$, and

$$
\left\{\begin{array}{l}
\left|X_{l}\right| \leq \bar{X} \\
\underline{p}_{i} \leq\left|p_{l, i}\right| \leq \bar{p}_{i} \\
0<\underline{q}_{i} \leq\left|q_{l, i}\right| \leq \bar{q}_{i}
\end{array}\right.
$$

\section{B. Self-Constructing Mechanism}

The approximation error in general depends on the number of fuzzy rules ( $N$ in this paper). A small number $N$ usually results in low accuracy. In contrast, the reduction of the approximation error becomes negligible if the number $N$ is adequately large. The role of self-constructing mechanism is to generate or delete rules in terms of the novelty of correction observation $\boldsymbol{z}(t)$ to the existing FBFs. With considerations analogous to $[34,38]$, a new rule is created when the distance between a new input signal and the current clusters is too far, while a redundant rule is removed when the fuzzy rule is insignificant. Note that the SCFNN starts with no fuzzy rule. Hence, $\boldsymbol{c}_{l}(0)=\emptyset, \boldsymbol{\sigma}_{l}(0)=\emptyset, \boldsymbol{W}_{l}(0)=\emptyset$, and $N(0)=0$, respectively. The system model presented in this study is continuous. However, the SCFNN needs sampled data to accomplish self-constructing. Thus, " $t-1$ " represents the previous sampling interval in the sequel [30,31]. Without loss of generality, suppose that there exist $N(t-1)$ FBFs to be adjusted before the current input $\boldsymbol{z}(t)$ arrives, i.e., $\boldsymbol{c}^{i}=$ $\left[c_{i}^{1}, c_{i}^{2}, \cdots, c_{i}^{N(t-1)}\right]^{T}, \quad \boldsymbol{\sigma}^{i}=\left[\sigma_{i}^{1}, \sigma_{i}^{2}, \cdots, \sigma_{i}^{N(t-1)}\right]^{T}, \quad i=$ $1,2, \cdots, m$, and $l=1,2, \cdots, N(t-1)$.
Define a measure between $\boldsymbol{z}(t)$ and the existing FBFs as:

$$
d_{l}=-\frac{\left(z(t)-c_{l}(t-1)\right)^{T}\left(z(t)-c_{l}(t-1)\right)}{\Sigma_{l}^{2}(t-1)},
$$

where $\boldsymbol{c}_{l}(t-1)=\left[c_{1}^{l(t-1)}, c_{2}^{l(t-1)}, \cdots, c_{m}^{l(t-1)}\right]^{T}, \mathbf{\Sigma}_{l}(t-1)=$ $\operatorname{diag}\left\{\sigma_{1}^{l(t-1)}, \sigma_{2}^{l(t-1)}, \cdots, \sigma_{m}^{l(t-1)}\right\}$, and $l=1,2, \cdots, N(t-1)$, respectively. The following is to present the criteria of rule generation and removal.

1) Rule Generation

Find the nearest fuzzy rule as:

$$
d_{\min }=\min _{l=1,2, \cdots, N(t-1)} d_{l}
$$

A new FBF is required:

$$
\left\{\begin{array}{l}
\boldsymbol{c}_{N}(t)=\boldsymbol{z}(t) \\
\boldsymbol{\sigma}_{N}(t)=\boldsymbol{\sigma}_{\mathrm{ini}} \\
\boldsymbol{W}_{N}(t)=0 \\
N(t)=N(t-1)+1
\end{array}\right.
$$

from the condition:

$$
d_{\min }>d_{t h}
$$

where $d_{t h}$ is a predefined threshold to be chosen as $d_{t h}=$ $\ln \left(1 / \epsilon_{1}\right), \boldsymbol{\sigma}_{\text {ini }}$ represents the initial width of the generated FBF, and $0<\epsilon_{1} \leq 1$, respectively.

2) Rule Removal

Find the redundant FBFs as:

$$
J_{r}=\left\{l^{\circ}\right\}, d_{l^{\circ}}<d_{0} .
$$

If the following condition satisfies:

$$
J_{r} \neq \varnothing,
$$

the redundant fuzzy rule is eliminated:

$$
\left\{\begin{array}{l}
\boldsymbol{c}_{l^{\circ}}=\varnothing \\
\boldsymbol{\sigma}_{l^{\circ}}=\varnothing \\
\boldsymbol{W}_{l^{\circ}}=\varnothing \\
N(t)=N(t)-\left|J_{r}\right|
\end{array},\right.
$$

where $d_{0}=\ln \left(1 / \epsilon_{0}\right)$ is a pre-specified threshold under which the fuzzy rule is determined inappropriate and $0<\epsilon_{0} \leq 1$, respectively.

For the sake of brevity, the self-constructing mechanism to produce or delete rules in terms of the novelty of correction observation $\boldsymbol{y}(t)$ to the existing FBFs is omitted herein.

Remark 5: The past few years have witnessed the development of learning approaches. A self-learning fuzzy logic system with reinforcement learning techniques can capture the desirability of states and adjust the fuzzy rules accordingly. One of the main hurdles is that the determination of fuzzy rules greatly relies on pure experiments [39, 40]. A 
radial basis function $\mathrm{NN}$ is integrated into a reinforcement learning paradigm for state value function approximation. One of the major challenges is "curse of dimensionality", which may limit wider applications to higher dimensional problems [41]. A self-organizing learning mechanism parameter is developed to adjust parameters. Nevertheless, the approximation accuracy decreases if inadequate fuzzy rules are prescribed [42]. In opposition to the aforementioned methods, the SCFNN is able to accomplish self-construction by properly creating and removing fuzzy rules, and to achieve accurate approximation. Moreover, fault identification plays an important role in an active FTAC system, affecting the fault-tolerance performance. Hence, SCFNN is integrated in the developed FTAC scheme, in order to accurately capture the upper bounds of the norms of the unknown terms.

\section{FAult-Tolerant Aircraft Control Design}

\section{A. An Overview of the Developed FTAC Scheme}

As depicted in Fig. 2, the FTAC system described here is mainly compounded by SCFNNs and finite-time fault-tolerant control, respectively. Within the proposed context, the SCFNNs with adaptive techniques serve to obtain online the values of $\hat{\rho}_{1}$ and $\hat{\rho}_{2}$. Based on the multivariable SMC approach and the SCFNN outputs, the FTAC law can thereby ensure the finitetime stability of the closed-loop system under unfavorable fault situations. It is worth mentioning that the FTAC is adapted in response to the information provided by the SCFNNs. In this sense, actuator faults can be accommodated in an active manner.

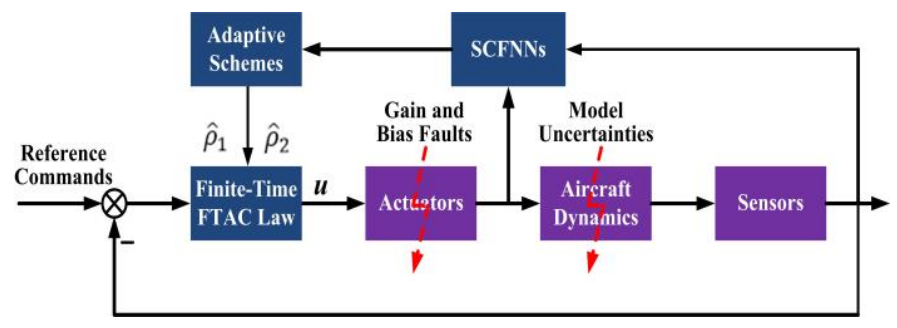

Fig. 2. Block diagram of the designed FTAC scheme.

\section{B. Analysis of SCFNN Approximation}

The upper bounds $\rho_{1}$ and $\rho_{2}$ are identified online by the SCFNNs, as indicated in Eqs. (27) and (28). The estimates, $\hat{\rho}_{1}$ and $\hat{\rho}_{2}$, are pivotal in the FTAC law design. A necessary step toward integrating the SCFNNs into the FTAC is the analysis of SCFNN approximation.

To proceed with the analysis, define the estimation errors as: $\widetilde{\boldsymbol{W}}=\boldsymbol{W}-\widehat{\boldsymbol{W}}, \widetilde{\boldsymbol{\phi}}=\boldsymbol{\phi}-\widehat{\boldsymbol{\phi}}, \tilde{\boldsymbol{c}}=\boldsymbol{c}-\hat{\boldsymbol{c}}$, and $\tilde{\boldsymbol{\sigma}}=\boldsymbol{\sigma}-\widehat{\boldsymbol{\sigma}}$, respectively. In accordance with Eq. (27) and Taylor series expansion, one can obtain:

$$
\begin{aligned}
\varepsilon_{1}= & \boldsymbol{W}^{T} \boldsymbol{\phi}-\widehat{\boldsymbol{W}}^{T} \widehat{\boldsymbol{\phi}} \\
= & \left(\boldsymbol{W}^{T} \boldsymbol{\phi}-\boldsymbol{W}^{T} \widehat{\boldsymbol{\phi}}-\widehat{\boldsymbol{W}}^{T} \boldsymbol{\phi}+\widehat{\boldsymbol{W}}^{T} \widehat{\boldsymbol{\phi}}\right) \\
& +\left(\boldsymbol{W}^{T} \widehat{\boldsymbol{\phi}}-\widehat{\boldsymbol{W}}^{T} \widehat{\boldsymbol{\phi}}\right)+\left(\widehat{\boldsymbol{W}}^{T} \boldsymbol{\phi}-\widehat{\boldsymbol{W}}^{T} \widehat{\boldsymbol{\phi}}\right) \\
= & \widetilde{\boldsymbol{W}}^{T} \widehat{\boldsymbol{\phi}}+\widehat{\boldsymbol{W}}^{T} \widetilde{\boldsymbol{\phi}}+\widetilde{\boldsymbol{W}}^{T} \widetilde{\boldsymbol{\phi}} \\
= & \widetilde{\boldsymbol{W}}^{T} \widehat{\boldsymbol{\phi}}+\widehat{\boldsymbol{W}}^{T}\left(\boldsymbol{\phi}_{c}^{\prime} \widetilde{\boldsymbol{c}}+\boldsymbol{\phi}_{\sigma}^{\prime} \widetilde{\boldsymbol{\sigma}}+\boldsymbol{h}(\mathbf{z} ; \widetilde{\boldsymbol{c}}, \widetilde{\boldsymbol{\sigma}})\right)+\widetilde{\boldsymbol{W}}^{T} \widetilde{\boldsymbol{\phi}} \\
= & \widetilde{\boldsymbol{W}}^{T} \widehat{\boldsymbol{\phi}}+\widehat{\boldsymbol{W}}^{T} \boldsymbol{\phi}_{\boldsymbol{c}}^{\prime} \tilde{\boldsymbol{c}}+\widehat{\boldsymbol{W}}^{T} \boldsymbol{\phi}_{\boldsymbol{\sigma}}^{\prime} \widetilde{\boldsymbol{\sigma}}+\underbrace{\widehat{W}^{T} \boldsymbol{h}(\mathbf{z} ; \widetilde{\boldsymbol{c}}, \widetilde{\boldsymbol{\sigma}})+\widetilde{\boldsymbol{W}}^{T} \widetilde{\boldsymbol{\phi}}}_{\varepsilon_{1}^{*}},
\end{aligned}
$$

where

$$
\begin{aligned}
\boldsymbol{\phi}_{\boldsymbol{c}}^{\prime} & =\frac{\partial \boldsymbol{\phi}}{\partial \boldsymbol{c}}=\operatorname{diag}\left(\boldsymbol{\phi}_{c_{1}}^{T}, \boldsymbol{\phi}_{c_{2}}^{T}, \cdots, \boldsymbol{\phi}_{c_{N}}^{T}\right) \in \mathcal{R}^{N \times m N}, \\
\boldsymbol{\phi}_{\boldsymbol{\sigma}}^{\prime} & =\frac{\partial \boldsymbol{\phi}}{\partial \boldsymbol{\sigma}}=\operatorname{diag}\left(\boldsymbol{\phi}_{\sigma_{1}}^{T}, \boldsymbol{\phi}_{\sigma_{2}}^{T}, \cdots, \boldsymbol{\phi}_{\sigma_{N}}^{T}\right) \in \mathcal{R}^{N \times m N}, \\
\boldsymbol{\phi}_{c_{l}}^{T} & =\frac{\partial \boldsymbol{\phi}_{l}}{\partial \boldsymbol{c}_{l}^{T}}=\left[\phi_{1, c_{l}}, \phi_{2, c_{l}}, \cdots, \phi_{m, c_{l}}\right] \\
& =2 \widehat{\boldsymbol{\phi}}_{l}\left[\frac{z_{1}-\hat{c}_{l, 1}}{\widehat{\sigma}_{l, 1}^{2}}, \frac{z_{2}-\hat{c}_{l, 2}}{\widehat{\sigma}_{l, 2}^{2}}, \cdots, \frac{z_{m}-\hat{c}_{l, m}}{\widehat{\sigma}_{l, m}^{2}}\right], \\
\boldsymbol{\phi}_{\boldsymbol{\sigma}_{l}}^{T} & =\frac{\partial \boldsymbol{\phi}_{l}}{\partial \boldsymbol{\sigma}_{l}^{T}}=\left[\phi_{1, \sigma_{l}}, \phi_{2, \sigma_{l}}, \cdots, \phi_{m, \sigma_{l}}\right] \\
& =2 \widehat{\boldsymbol{\phi}}_{l}\left[\frac{\left(z_{1}-\hat{c}_{l, 1}\right)^{2}}{\widehat{\sigma}_{l, 1}^{3}}, \frac{\left(z_{2}-\hat{c}_{l, 2}\right)^{2}}{\widehat{\sigma}_{l, 2}^{3}}, \cdots, \frac{\left(z_{m}-\hat{c}_{l, m}\right)^{2}}{\widehat{\sigma}_{l, m}^{3}}\right]
\end{aligned}
$$

and $\boldsymbol{h}(\mathbf{z} ; \tilde{\boldsymbol{c}}, \widetilde{\boldsymbol{\sigma}})=\boldsymbol{\phi}-\widehat{\boldsymbol{\phi}}-\boldsymbol{\phi}_{c}^{\prime} \tilde{\boldsymbol{c}}-\boldsymbol{\phi}_{\sigma}^{\prime} \widetilde{\boldsymbol{\sigma}}$ represents a high order term.

In Eq. (38), $\varepsilon_{1}^{*}=\widehat{\boldsymbol{W}}^{T} \boldsymbol{h}(\mathbf{z} ; \tilde{\boldsymbol{c}}, \widetilde{\boldsymbol{\sigma}})+\widetilde{\boldsymbol{W}}^{T} \widetilde{\boldsymbol{\phi}}$ is thought as the residual approximation error. The analysis of $\varepsilon_{1}^{*}$ is then conducted as:

$$
\begin{aligned}
& \begin{array}{c}
\left(\varepsilon_{1}^{*}\right)^{T} \varepsilon_{1}^{*}=\left[\widehat{\boldsymbol{W}}^{T}\left(\widetilde{\boldsymbol{\phi}}-\boldsymbol{\phi}_{c}^{\prime} \tilde{\boldsymbol{c}}-\boldsymbol{\phi}_{\sigma}^{\prime} \widetilde{\boldsymbol{\sigma}}\right)+\widetilde{\boldsymbol{W}}^{T} \widetilde{\boldsymbol{\phi}}\right]^{T}\left[\widehat { \boldsymbol { W } } ^ { T } \left(\widetilde{\boldsymbol{\phi}}-\boldsymbol{\phi}_{c}^{\prime} \tilde{\boldsymbol{c}}\right.\right. \\
\left.\left.-\boldsymbol{\phi}_{\boldsymbol{\sigma}}^{\prime} \widetilde{\boldsymbol{\sigma}}\right)+\widetilde{\boldsymbol{W}}^{T} \widetilde{\boldsymbol{\phi}}\right]
\end{array} \\
& =\widetilde{\boldsymbol{\phi}}^{T} \widehat{\boldsymbol{W}} \widehat{\boldsymbol{W}}^{T} \widetilde{\boldsymbol{\phi}}+\tilde{\boldsymbol{c}}^{T}\left(\boldsymbol{\phi}_{c}^{\prime}\right)^{T} \widehat{\boldsymbol{W}} \widehat{\boldsymbol{W}}^{T} \boldsymbol{\phi}_{c}^{\prime} \tilde{\boldsymbol{c}}+\widetilde{\boldsymbol{\sigma}}^{T}\left(\boldsymbol{\phi}_{\boldsymbol{\sigma}}^{\prime}\right)^{T} \widehat{\boldsymbol{W}} \widehat{\boldsymbol{W}}^{T} \boldsymbol{\phi}_{\boldsymbol{\sigma}}^{\prime} \widetilde{\boldsymbol{\sigma}} \\
& -2 \tilde{\boldsymbol{c}}^{T}\left(\boldsymbol{\phi}_{c}^{\prime}\right)^{T} \widehat{\boldsymbol{W}} \boldsymbol{W}^{T} \widetilde{\boldsymbol{\phi}}-2 \widetilde{\boldsymbol{\sigma}}^{T}\left(\boldsymbol{\phi}_{\boldsymbol{\sigma}}^{\prime}\right)^{T} \widehat{\boldsymbol{W}} \boldsymbol{W}^{T} \widetilde{\boldsymbol{\phi}} \\
& +2 \widetilde{\boldsymbol{\sigma}}^{T}\left(\boldsymbol{\phi}_{\boldsymbol{\sigma}}^{\prime}\right)^{T} \widehat{\boldsymbol{W}} \widehat{\boldsymbol{W}}^{T} \boldsymbol{\phi}_{\boldsymbol{c}}^{\prime} \tilde{\boldsymbol{c}}+\widetilde{\boldsymbol{\phi}}^{T} \widetilde{\boldsymbol{W}} \widetilde{\boldsymbol{W}}^{T} \widetilde{\boldsymbol{\phi}}+2 \widetilde{\boldsymbol{\phi}}^{T} \widehat{\boldsymbol{W}} \widetilde{\boldsymbol{W}}^{T} \widetilde{\boldsymbol{\phi}} .
\end{aligned}
$$

Moreover, the following inequalities hold:

$$
\left\{\begin{array}{l}
2 \widetilde{\boldsymbol{\phi}}^{T} \widehat{\boldsymbol{W}} \widetilde{\boldsymbol{W}}^{T} \widetilde{\boldsymbol{\phi}} \leq \widetilde{\boldsymbol{\phi}}^{T} \widehat{\boldsymbol{W}} \widehat{\boldsymbol{W}}^{T} \widetilde{\boldsymbol{\phi}}+\widetilde{\boldsymbol{\phi}}^{T} \widetilde{\boldsymbol{W}} \widetilde{\boldsymbol{W}}^{T} \widetilde{\boldsymbol{\phi}} \\
2 \widetilde{\boldsymbol{\sigma}}^{T}\left(\boldsymbol{\phi}_{\boldsymbol{\sigma}}^{\prime}\right)^{T} \widehat{\boldsymbol{W}} \boldsymbol{W}^{T} \boldsymbol{\phi}_{c}^{\prime} \tilde{\boldsymbol{c}} \leq \tilde{\boldsymbol{c}}^{T}\left(\boldsymbol{\phi}_{\boldsymbol{c}}^{\prime}\right)^{T} \widehat{\boldsymbol{W}} \widehat{\boldsymbol{W}}^{T} \boldsymbol{\phi}_{\boldsymbol{c}}^{\prime} \tilde{\boldsymbol{c}}+\widetilde{\boldsymbol{\sigma}}^{T}\left(\boldsymbol{\phi}_{\boldsymbol{\sigma}}^{\prime}\right)^{T} \widehat{\boldsymbol{W}} \widehat{\boldsymbol{W}}^{T} \boldsymbol{\phi}_{\boldsymbol{\sigma}}^{\prime} \widetilde{\boldsymbol{\sigma}} \\
-2 \tilde{\boldsymbol{c}}^{T}\left(\boldsymbol{\phi}_{c}^{\prime}\right)^{T} \widehat{\boldsymbol{W}} \boldsymbol{W}^{T} \widetilde{\boldsymbol{\phi}} \leq \tilde{\boldsymbol{c}}^{T}\left(\boldsymbol{\phi}_{c}^{\prime}\right)^{T} \widehat{\boldsymbol{W}} \widehat{\boldsymbol{W}}^{T} \boldsymbol{\phi}_{c}^{\prime} \tilde{\boldsymbol{c}}+\widetilde{\boldsymbol{\phi}}^{T} \boldsymbol{W} \boldsymbol{W}^{T} \widetilde{\boldsymbol{\phi}} \\
-2 \widetilde{\boldsymbol{\sigma}}^{T}\left(\boldsymbol{\phi}_{\boldsymbol{\sigma}}^{\prime}\right)^{T} \widehat{\boldsymbol{W}} \boldsymbol{W}^{T} \widetilde{\boldsymbol{\phi}} \leq \widetilde{\boldsymbol{\sigma}}^{T}\left(\boldsymbol{\phi}_{\boldsymbol{\sigma}}^{\prime}\right)^{T} \widehat{\boldsymbol{W}} \widehat{\boldsymbol{W}}^{T} \boldsymbol{\phi}_{\boldsymbol{\sigma}}^{\prime} \widetilde{\boldsymbol{\sigma}}+\widetilde{\boldsymbol{\phi}}^{T} \boldsymbol{W} \boldsymbol{W}^{T} \widetilde{\boldsymbol{\phi}}
\end{array}\right.
$$

Hence, applying (44) to Eq. (43) results in:

$$
\begin{aligned}
\left(\varepsilon_{1}^{*}\right)^{T} \varepsilon_{1}^{*} \leq & 3 \tilde{\boldsymbol{c}}^{T}\left(\boldsymbol{\phi}_{\boldsymbol{c}}^{\prime}\right)^{T} \widehat{\boldsymbol{W}} \widehat{\boldsymbol{W}}^{T} \boldsymbol{\phi}_{c}^{\prime} \tilde{\boldsymbol{c}}+3 \widetilde{\boldsymbol{\sigma}}^{T}\left(\boldsymbol{\phi}_{\boldsymbol{\sigma}}^{\prime}\right)^{T} \widehat{\boldsymbol{W}} \widehat{\boldsymbol{W}}^{T} \boldsymbol{\phi}_{\boldsymbol{\sigma}}^{\prime} \widetilde{\boldsymbol{\sigma}} \\
& +2 \widetilde{\boldsymbol{\phi}}^{T} \widetilde{\boldsymbol{W}} \widetilde{\boldsymbol{W}}^{T} \widetilde{\boldsymbol{\phi}}+2 \widetilde{\boldsymbol{\phi}}^{T} \widehat{\boldsymbol{W}} \widehat{\boldsymbol{W}}^{T} \widetilde{\boldsymbol{\phi}}+2 \widetilde{\boldsymbol{\phi}}^{T} \boldsymbol{W} \boldsymbol{W}^{T} \widetilde{\boldsymbol{\phi}}
\end{aligned}
$$

Given that $0<\|\widetilde{\boldsymbol{\phi}}\| \leq 1$ and $d_{0}>\frac{\left(\mathbf{z}-\boldsymbol{c}_{l}\right)^{T}\left(\mathbf{z}-\boldsymbol{c}_{l}\right)}{\Sigma_{l}^{2}}, l=1,2, \cdots, N$, the terms of the right hand side of (45) are individually analyzed:

$$
\begin{gathered}
\lambda_{\max }\left[\left(\boldsymbol{\phi}_{\boldsymbol{c}}^{\prime}\right)^{T} \widehat{\boldsymbol{W}} \widehat{\boldsymbol{W}}^{T} \boldsymbol{\phi}_{\boldsymbol{c}}^{\prime}\right] \\
\leq \bar{W}^{2} \lambda_{\max }\left[\boldsymbol{\phi}_{\boldsymbol{c}_{1}} \boldsymbol{\phi}_{\boldsymbol{c}_{1}}^{T}, \boldsymbol{\phi}_{\boldsymbol{c}_{2}} \boldsymbol{\phi}_{\boldsymbol{c}_{2}}^{T}, \cdots, \boldsymbol{\phi}_{\boldsymbol{c}_{N}} \boldsymbol{\phi}_{\boldsymbol{c}_{N}}^{T}\right] \leq \frac{2 d_{0} \bar{W}^{2}}{\underline{\sigma}^{2}}, \\
\lambda_{\max }\left[\left(\boldsymbol{\phi}_{\boldsymbol{\sigma}}^{\prime}\right)^{T} \widehat{\boldsymbol{W}} \widehat{\boldsymbol{W}}^{T} \boldsymbol{\phi}_{\boldsymbol{\sigma}}^{\prime}\right] \\
\leq \bar{W}^{2} \lambda_{\max }\left[\boldsymbol{\phi}_{\boldsymbol{\sigma}_{1}} \boldsymbol{\phi}_{\boldsymbol{\sigma}_{1}}^{T}, \boldsymbol{\phi}_{\boldsymbol{\sigma}_{2}} \boldsymbol{\phi}_{\boldsymbol{\sigma}_{2}}^{T}, \cdots, \boldsymbol{\phi}_{\boldsymbol{\sigma}_{N}} \boldsymbol{\phi}_{\boldsymbol{\sigma}_{N}}^{T}\right] \leq \frac{2 d_{0}^{2} \bar{W}^{2}}{\underline{\sigma}^{2}}, \\
2 \widetilde{\boldsymbol{\phi}}^{T} \widetilde{\boldsymbol{W}} \widetilde{\boldsymbol{W}}^{T} \widetilde{\boldsymbol{\phi}} \leq 2 \operatorname{trace}\left(\widetilde{\boldsymbol{W}} \widetilde{\boldsymbol{W}}^{T}\right), \\
2 \widetilde{\boldsymbol{\phi}}^{T} \widehat{\boldsymbol{W}} \widehat{\boldsymbol{W}}^{T} \widetilde{\boldsymbol{\phi}}+2 \widetilde{\boldsymbol{\phi}}^{T} \boldsymbol{W} \boldsymbol{W}^{T} \widetilde{\boldsymbol{\phi}} \leq 4 \bar{W}^{2} .
\end{gathered}
$$

In consequence, one can achieve:

$$
\left(\varepsilon_{1}^{*}\right)^{T} \varepsilon_{1}^{*} \leq \frac{6 d_{0} \bar{W}^{2}}{\underline{\sigma}^{2}}+\frac{6 d_{0}^{2} \bar{W}^{2}}{\underline{\sigma}^{2}}+2 \operatorname{trace}\left(\widetilde{\boldsymbol{W}} \widetilde{\boldsymbol{W}}^{T}\right)+4 \bar{W}^{2}<\infty,
$$


which implies that the residual approximation error is bounded.

By performing the similar analysis, it can be achieved that $\varepsilon_{2}^{*}=\widetilde{\boldsymbol{X}}^{T} \boldsymbol{h}(\boldsymbol{z} ; \widetilde{\boldsymbol{p}}, \widetilde{\boldsymbol{q}})+\widetilde{\boldsymbol{X}}^{T} \widetilde{\boldsymbol{\psi}}$ is bounded as well, where $\widetilde{\boldsymbol{X}}=\boldsymbol{X}-$ $\widehat{\boldsymbol{X}}, \widetilde{\boldsymbol{\psi}}=\boldsymbol{\psi}-\widehat{\boldsymbol{\psi}}, \widetilde{\boldsymbol{p}}=\boldsymbol{p}-\widehat{\boldsymbol{p}}, \widetilde{\boldsymbol{q}}=\boldsymbol{q}-\widehat{\boldsymbol{q}}$, and $\boldsymbol{h}(\boldsymbol{z} ; \widetilde{\boldsymbol{p}}, \widetilde{\boldsymbol{q}})$ denotes a high order term, respectively.

\section{The Proposed FTAC Algorithm}

Define the error vector as: $\boldsymbol{e}=\boldsymbol{x}-\boldsymbol{x}_{d}$, where $\boldsymbol{x}_{d}$ represents the command vector. In order to achieve a sliding mode throughout the entire system response, the sliding manifold is established as:

$$
\boldsymbol{S}=\boldsymbol{e}+\mu \int_{0}^{t}\left(\|\boldsymbol{e}\|^{r} \boldsymbol{e} /\|\boldsymbol{e}\|\right) d \tau,
$$

where $\mu$ is a positive constant and $0<r<1$, respectively. The goal of FTAC pursued in this study is to steer the tracking error vector $\boldsymbol{e}$ to the origin along $\boldsymbol{S}=0$ in finite time, under actuator faults and model uncertainties. The following Theorem 1 constitutes the main result of FTAC design.

Theorem 1: Given the faulty model of aircraft in Eq. (20), the FTAC law is constructed as:

$$
\boldsymbol{u}=-\boldsymbol{g}_{0}^{+}\left[\boldsymbol{F}+\left(\hat{\rho}_{1}+\hat{\rho}_{2}\|\boldsymbol{F}\|+\eta\right) \frac{\boldsymbol{s}}{\|\boldsymbol{S}\|}\right]
$$

where $\quad \boldsymbol{F}=\boldsymbol{f}_{0}-\dot{\boldsymbol{x}}_{d}+\mu\left(\|\boldsymbol{e}\|^{r} \boldsymbol{e} /\|\boldsymbol{e}\|\right) \quad, \quad \eta \geq$ $\frac{\left(\widehat{\rho}_{1} \widehat{\rho}_{2}+\widehat{\rho}_{1} \varepsilon_{2}^{*}+\varepsilon_{1}^{*}\right)+\left(\widehat{\rho}_{2} \rho_{2}+\widehat{\rho}_{2} \varepsilon_{2}^{*}+\varepsilon_{2}^{*}\right)\|\boldsymbol{F}\|}{1-\widehat{\rho}_{2}-\varepsilon_{2}^{*}}+\varepsilon_{\eta}, \hat{\rho}_{1}=\widehat{\boldsymbol{W}}^{T} \widehat{\boldsymbol{\phi}}, \hat{\rho}_{2}=\widehat{\boldsymbol{X}}^{T} \widehat{\boldsymbol{\psi}}$, and $\varepsilon_{\eta}>0$, respectively. Moreover, the adaptation laws are formed as:

$$
\begin{aligned}
& \dot{\widehat{W}}=\widehat{\boldsymbol{\phi}} \\
& \dot{\hat{c}}_{l, i}=\phi_{i, c_{l}} \widehat{W}_{l}^{T} \text {, } \\
& \dot{\hat{\sigma}}_{l, i}=\phi_{i, \sigma_{l}} \widehat{W}_{l}^{T}, \\
& \dot{\hat{\boldsymbol{X}}}=\hat{\rho}_{1} \widehat{\boldsymbol{\psi}}+\widehat{\boldsymbol{\psi}}\|\boldsymbol{F}\|+\hat{\rho}_{2} \widehat{\boldsymbol{\psi}}\|\boldsymbol{F}\|+\eta \widehat{\boldsymbol{\psi}}, \\
& \dot{\hat{p}}_{l, i}=\psi_{i, p_{l}} \hat{X}_{l}^{T}\|\boldsymbol{F}\|+\hat{\rho}_{1} \psi_{i, p_{l}} \hat{X}_{l}^{T}+\hat{\rho}_{2} \psi_{i, p_{l}} \hat{X}_{l}^{T}\|\boldsymbol{F}\|+\eta \psi_{i, p_{l}} \hat{X}_{l}^{T} \text {, } \\
& \dot{\hat{q}}_{l, i}=\psi_{i, q_{l}} \hat{X}_{l}^{T}\|\boldsymbol{F}\|+\hat{\rho}_{1} \psi_{i, q_{l}} \hat{X}_{l}^{T}+\hat{\rho}_{2} \psi_{i, q_{l}} \hat{X}_{l}^{T}\|\boldsymbol{F}\|+\eta \psi_{i, q_{l}} \hat{X}_{l}^{T},
\end{aligned}
$$

where $i=1,2, \cdots, m$ and $l=1,2, \cdots, N$. Therefore, the designed FTAC law ensures that the tracking error $\boldsymbol{e}$ can be enforced to the origin along $\boldsymbol{S}=0$ in finite time even if subject to actuator malfunctions and model uncertainties.

Proof:

Choose a Lyapunov function candidate as:

$$
\begin{aligned}
V= & \underbrace{\|\boldsymbol{S}\|}_{V_{0}}+\underbrace{\frac{1}{2} \operatorname{trace}\left(\widetilde{\boldsymbol{W}}^{T} \widetilde{\boldsymbol{W}}\right)+\frac{1}{2} \tilde{\boldsymbol{c}}^{T} \widetilde{\boldsymbol{c}}+\frac{1}{2} \widetilde{\boldsymbol{\sigma}}^{T} \widetilde{\boldsymbol{\sigma}}}_{V_{2}} \\
& +\underbrace{\frac{1}{2} \operatorname{trace}\left(\widetilde{\boldsymbol{X}}^{T} \widetilde{\boldsymbol{X}}\right)+\frac{1}{2} \widetilde{\boldsymbol{p}}^{T} \widetilde{\boldsymbol{p}}+\frac{1}{2} \widetilde{\boldsymbol{q}}^{T} \widetilde{\boldsymbol{q}}}_{V_{1}} .
\end{aligned}
$$

For simplicity, the proof procedure is divided into four steps. $V_{0}, V_{1}$, and $V_{2}$ are differentiated in sequence. At the last step, the results of the first three steps are combined.

Step 1) Differentiating $V_{0}$ gives:

$$
\begin{aligned}
& \dot{V}_{0}=\frac{S^{T}}{\|S\|} \dot{S} \\
& =\frac{\boldsymbol{S}^{T}}{\|\boldsymbol{S}\|}\left[\left(\boldsymbol{f}_{0}+\boldsymbol{g}_{0} \boldsymbol{u}\right)+\left(\boldsymbol{\Delta}_{f}+\left(\boldsymbol{g}_{0}+\boldsymbol{\Delta}_{g}\right) \mathbf{Y}\right)\right. \\
& \left.+\left(\boldsymbol{g}_{0}(\boldsymbol{\Lambda}-\boldsymbol{I})+\boldsymbol{\Delta}_{g} \boldsymbol{\Lambda}\right) \boldsymbol{u}+\mu\left(\|\boldsymbol{e}\|^{r} \boldsymbol{e} /\|\boldsymbol{e}\|\right)-\dot{\boldsymbol{x}}_{d}\right] \\
& \leq\left(\rho_{1}-\hat{\rho}_{1}+\hat{\rho}_{1} \rho_{2}\right)+\left(\rho_{2}-\hat{\rho}_{2}+\hat{\rho}_{2} \rho_{2}\right)\|\boldsymbol{F}\|+\left(-\eta+\eta \rho_{2}\right) \\
& =\underbrace{\left(\rho_{1}-\hat{\rho}_{1}\right)}_{\text {Term } 1}+\underbrace{\hat{\rho}_{1} \rho_{2}+\left(\rho_{2}-\hat{\rho}_{2}+\hat{\rho}_{2} \rho_{2}\right)\|\boldsymbol{F}\|+\eta \rho_{2}}_{\text {Term } 2} \underbrace{-\eta}_{\text {Term } 3} \text {. }
\end{aligned}
$$

Step 2) Differentiating $V_{1}$ renders:

$$
\begin{aligned}
\dot{V}_{1} & =-\widetilde{\boldsymbol{W}}^{T} \dot{\boldsymbol{W}}-\tilde{\boldsymbol{c}}^{T} \dot{\hat{\boldsymbol{c}}}-\widetilde{\boldsymbol{\sigma}}^{T} \dot{\hat{\boldsymbol{\sigma}}} \\
& =-\widetilde{\boldsymbol{W}}^{T} \widehat{\boldsymbol{\phi}}-\tilde{\boldsymbol{c}}^{T}\left(\boldsymbol{\phi}_{\boldsymbol{c}}^{\prime}\right)^{T} \widehat{\boldsymbol{W}}-\widetilde{\boldsymbol{\sigma}}^{T}\left(\boldsymbol{\phi}_{\boldsymbol{\sigma}}^{\prime}\right)^{T} \widehat{\boldsymbol{W}} .
\end{aligned}
$$

In addition, the first term of right hand side of (60) plus $\dot{V}_{1}$ is:

$$
\operatorname{Term} 1+\dot{V}_{1} \leq \varepsilon_{1}^{*}
$$

Step 3) Differentiating $V_{2}$ leads to:

$$
\begin{aligned}
\dot{V}_{2}= & -\widetilde{\boldsymbol{X}}^{T} \dot{\hat{\boldsymbol{X}}}-\widetilde{\boldsymbol{p}}^{T} \dot{\hat{\boldsymbol{p}}}-\widetilde{\boldsymbol{q}}^{T} \dot{\boldsymbol{\boldsymbol { q }}}^{-} \\
= & -\widetilde{\boldsymbol{X}}^{T} \hat{\rho}_{1} \widehat{\boldsymbol{\psi}}-\widetilde{\boldsymbol{X}}^{T} \widehat{\boldsymbol{\psi}}\|\boldsymbol{F}\|-\widetilde{\boldsymbol{X}}^{T} \hat{\rho}_{2} \widehat{\boldsymbol{\psi}}\|\boldsymbol{F}\|-\widetilde{\boldsymbol{X}}^{T} \eta \widehat{\boldsymbol{\psi}} \\
& -\widetilde{\boldsymbol{p}}^{T}\left(\boldsymbol{\phi}_{\boldsymbol{p}}^{\prime}\right)^{T} \widehat{\boldsymbol{X}}\|\boldsymbol{F}\|-\widetilde{\boldsymbol{p}}^{T} \hat{\rho}_{1}\left(\boldsymbol{\phi}_{\boldsymbol{p}}^{\prime}\right)^{T} \widehat{\boldsymbol{X}} \\
& -\widetilde{\boldsymbol{p}}^{T} \hat{\rho}_{2}\left(\boldsymbol{\phi}_{\boldsymbol{p}}^{\prime}\right)^{T} \widehat{\boldsymbol{X}}\|\boldsymbol{F}\|-\widetilde{\boldsymbol{p}}^{T} \eta\left(\boldsymbol{\phi}_{\boldsymbol{p}}^{\prime}\right)^{T} \widehat{\boldsymbol{X}} \\
& -\widetilde{\boldsymbol{q}}^{T}\left(\boldsymbol{\phi}_{\boldsymbol{q}}^{\prime}\right)^{T} \widehat{\boldsymbol{X}}\|\boldsymbol{F}\|-\widetilde{\boldsymbol{q}}^{T} \hat{\rho}_{1}\left(\boldsymbol{\phi}_{\boldsymbol{q}}^{\prime}\right)^{T} \widehat{\boldsymbol{X}} \\
& -\widetilde{\boldsymbol{q}}^{T} \hat{\rho}_{2}\left(\boldsymbol{\phi}_{\boldsymbol{q}}^{\prime}\right)^{T} \widehat{\boldsymbol{X}}\|\boldsymbol{F}\|-\widetilde{\boldsymbol{q}}^{T} \eta\left(\boldsymbol{\phi}_{q}^{\prime}\right)^{T} \widehat{\boldsymbol{X}} \\
= & -\hat{\rho}_{1}\left(\widetilde{\boldsymbol{X}}^{T} \widehat{\boldsymbol{\psi}}+\widehat{\boldsymbol{X}}^{T} \boldsymbol{\phi}_{p}^{\prime} \widetilde{\boldsymbol{p}}^{\prime}+\widehat{\boldsymbol{X}}^{T} \boldsymbol{\phi}_{\boldsymbol{q}}^{\prime} \widetilde{\boldsymbol{q}}\right) \\
& -\left(\widetilde{\boldsymbol{X}}^{T} \widehat{\boldsymbol{\psi}}+\widehat{\boldsymbol{X}}^{T} \boldsymbol{\phi}_{\boldsymbol{p}}^{\prime} \widetilde{\boldsymbol{p}}+\widehat{\boldsymbol{X}}^{T} \boldsymbol{\phi}_{\boldsymbol{q}}^{\prime} \widetilde{\boldsymbol{q}}\right)\|\boldsymbol{F}\| \\
& -\hat{\rho}_{2}\left(\widetilde{\boldsymbol{X}}^{T} \widehat{\boldsymbol{\psi}}+\widehat{\boldsymbol{X}}^{T} \boldsymbol{\phi}_{\boldsymbol{p}}^{\prime} \widetilde{\boldsymbol{p}}+\widehat{\boldsymbol{X}}^{T} \boldsymbol{\phi}_{q}^{\prime} \widetilde{\boldsymbol{q}}\right)\|\boldsymbol{F}\| \\
& -\eta\left(\widetilde{\boldsymbol{X}}^{T} \widehat{\boldsymbol{\psi}}+\widehat{\boldsymbol{X}}^{T} \boldsymbol{\phi}_{p}^{\prime} \widetilde{\boldsymbol{p}}+\widehat{\boldsymbol{X}}^{T} \boldsymbol{\phi}_{q}^{\prime} \widetilde{\boldsymbol{q}}\right) .
\end{aligned}
$$

Since $\varepsilon_{2}=\rho_{2}-\hat{\rho}_{2}=\widetilde{\boldsymbol{X}}^{T} \widehat{\boldsymbol{\psi}}+\widehat{\boldsymbol{X}}^{T} \boldsymbol{\phi}_{p}^{\prime} \widetilde{\boldsymbol{p}}+\widehat{\boldsymbol{X}}^{T} \boldsymbol{\phi}_{q}^{\prime} \widetilde{\boldsymbol{q}}+\varepsilon_{2}^{*}$,

$$
\begin{aligned}
\dot{V}_{2} \leq & -\hat{\rho}_{1}\left(\rho_{2}-\hat{\rho}_{2}-\varepsilon_{2}^{*}\right)-\left(\rho_{2}-\hat{\rho}_{2}-\varepsilon_{2}^{*}\right)\|\boldsymbol{F}\| \\
& -\hat{\rho}_{2}\left(\rho_{2}-\hat{\rho}_{2}-\varepsilon_{2}^{*}\right)\|\boldsymbol{F}\|-\eta\left(\rho_{2}-\hat{\rho}_{2}-\varepsilon_{2}^{*}\right) .
\end{aligned}
$$

Consequently, the second term of right hand side of (60) plus $\dot{V}_{2}$ can render:

$$
\begin{aligned}
\text { Term } 2+\dot{V}_{2} \leq & \left(\hat{\rho}_{1} \rho_{2}-\hat{\rho}_{1} \rho_{2}+\hat{\rho}_{1} \hat{\rho}_{2}+\hat{\rho}_{1} \varepsilon_{2}^{*}\right)+\varepsilon_{2}^{*}\|\boldsymbol{F}\| \\
& +\left(-\hat{\rho}_{2} \rho_{2}+\hat{\rho}_{2} \hat{\rho}_{2}+\hat{\rho}_{2} \rho_{2}+\hat{\rho}_{2} \varepsilon_{2}^{*}\right)\|\boldsymbol{F}\| \\
& +\left(\eta \rho_{2}-\eta \rho_{2}+\eta \hat{\rho}_{2}+\eta \varepsilon_{2}^{*}\right) \\
= & \left(\hat{\rho}_{1} \hat{\rho}_{2}+\hat{\rho}_{1} \varepsilon_{2}^{*}\right)+\varepsilon_{2}^{*}\|\boldsymbol{F}\| \\
& +\left(\hat{\rho}_{2} \rho_{2}+\hat{\rho}_{2} \varepsilon_{2}^{*}\right)\|\boldsymbol{F}\|+\left(\eta \hat{\rho}_{2}+\eta \varepsilon_{2}^{*}\right) .
\end{aligned}
$$

Step 4) By recalling the results in (60), (62), and (65), $\dot{V}$ can be further bounded as:

$$
\begin{aligned}
\dot{V}= & \dot{V}_{0}+\dot{V}_{1}+\dot{V}_{2} \\
\leq & -\eta+\left(\hat{\rho}_{1} \hat{\rho}_{2}+\hat{\rho}_{1} \varepsilon_{2}^{*}+\varepsilon_{1}^{*}\right) \\
& +\left(\hat{\rho}_{2} \rho_{2}+\hat{\rho}_{2} \varepsilon_{2}^{*}+\varepsilon_{2}^{*}\right)\|\boldsymbol{F}\|+\eta\left(\hat{\rho}_{2}+\varepsilon_{2}^{*}\right) .
\end{aligned}
$$

If $\varepsilon_{\eta}>0$ and the condition:

$$
\eta \geq \frac{\left(\widehat{\rho}_{1} \widehat{\rho}_{2}+\widehat{\rho}_{1} \varepsilon_{2}^{*}+\varepsilon_{1}^{*}\right)+\left(\widehat{\rho}_{2} \rho_{2}+\widehat{\rho}_{2} \varepsilon_{2}^{*}+\varepsilon_{2}^{*}\right)\|\boldsymbol{F}\|}{1-\widehat{\rho}_{2}-\varepsilon_{2}^{*}}+\varepsilon_{\eta},
$$


holds, the following inequality:

$$
\dot{V}=\dot{V}_{0}+\dot{V}_{1}+\dot{V}_{2} \leq-\varepsilon_{\eta},
$$

can be guaranteed.

According to [43], the condition (68) implies that the values of $V$ and $\boldsymbol{S}$ will converge to zero in finite time $t_{f}$, i.e., $V\left(t_{f}\right)=$ 0 . One can obtain that $t_{f} \leq t_{0}+\frac{V\left(t_{0}\right)}{\varepsilon_{\eta}}$. In addition, since the value of $V$ is bounded, $\widetilde{\boldsymbol{W}}, \widetilde{\boldsymbol{c}}, \widetilde{\boldsymbol{\sigma}}, \widetilde{\boldsymbol{X}}, \widetilde{\boldsymbol{p}}$, and $\widetilde{\boldsymbol{q}}$ are all bounded. This completes the proof.

Remark 6: As indicated in Eq. (52), the FTAC law is designed to compensate for the detrimental impact arising from actuator faults and model uncertainties. Notice that the estimates of $\boldsymbol{W}, \boldsymbol{\phi}, \boldsymbol{X}$, and $\boldsymbol{\psi}$ can be gained by resorting to the adaptation algorithms. Thus, the values of $\rho_{1}$ and $\rho_{2}$ can be adapted in response to the up-to-date knowledge from SCFNNs.

Remark 7: The typical SMC design can be divided into two steps. In the first step, a sliding manifold is established, such that the system trajectory along the manifold acquires the desired specifications. In the second step, the goal pursued is to guarantee that the system trajectory can reach the manifold in finite time. As can be observed from Eq. (51), the sliding manifold is established by vector expression, which can be named multivariable design [44]. The resulting FTAC law, as shown in Eq. (52), is integrated by multivariable design as well. In most of the existing sliding mode based FTAC approaches, the design problem is typically formulated as the problems involving single input control channel. In contrast, the approach of this work yields a multivariable design to be considered in FTAC, avoiding decoupled issues.

Remark 8: Focusing on the FTAC strategy (52), there are four important design parameters $\left(\mu, \hat{\rho}_{1}, \hat{\rho}_{2}, \eta\right)$. To be more specific, $\hat{\rho}_{1}$ and $\hat{\rho}_{2}$ can be obtained by the adaptive laws (53)(58). The design parameter $\mu$ in the sliding manifold is closely related to the sliding speed when $\boldsymbol{S}=0$. The parameter $\eta$ affects the convergence rate of reaching sliding surface (from $\boldsymbol{S} \neq 0$ to $\boldsymbol{S}=0$ ). However, if $\eta$ is too large, chattering phenomena will be induced. In the existing literature, to the best of the authors' knowledge, there is no systematic method to choose the values of the parameters. In this study, the values are selected by the extensive tests and trials.

\section{NUMERICAL SIMULATIONS}

\section{A. Simulation Scenarios}

The trimming flight conditions of the aircraft are: $\alpha_{\text {trim }}=$ $0.928^{\circ}, q_{\text {trim }}=0, V_{\text {trim }}=230 \mathrm{~m} / \mathrm{s}, h_{\text {trim }}=7000 \mathrm{~m}$, $\theta_{\text {trim }}=0.928^{\circ}, \delta_{e, \text { trim }}=0, \delta_{s, \text { trim }}=0.733^{\circ}$, and $T_{\text {trim }}=$ $41631 \mathrm{~N}$, respectively. $\mu=15$ and $\eta=2$. The estimated values of $\rho_{1}$ and $\rho_{2}$ are illustrated in Fig. 6 .

The factors involving the model uncertainties, actuator faults, and noises in measurement channels are taken into consideration in the simulations.

- Factor 1: The mass of the aircraft is perturbed by $20 \%$ of its nominal value. The perturbation corresponding to $I_{y y}$ is $20 \%$ of the nominal value. The maximal $20 \%$ mismatch is present in $\bar{c}, \bar{q}$, and $S_{r}$.
- Factor 2: The gain and bias faults in the elevator and the thrust are:

$$
\begin{aligned}
& \lambda_{1}=\left\{\begin{array}{lr}
1, & 0 \leq t<7 \\
0, & t \geq 7
\end{array}, \sigma_{1}=\left\{\begin{array}{lr}
0, & 0 \leq t<7 \\
6, & t \geq 7
\end{array},\right.\right. \\
& \lambda_{2}=\left\{\begin{array}{lr}
1, & 0 \leq t<7 \\
0.5, & t \geq 7
\end{array}, \sigma_{2}=\left\{\begin{array}{lr}
0, & 0 \leq t<7 \\
2, & t \geq 7
\end{array} .\right.\right.
\end{aligned}
$$

- Factor 3: The white noise with a mean of 0 and covariance of 0.01 is injected into each of measurement channels.

Furthermore, in order to quantitatively evaluate the tracking performance, define:

$$
\left\{\begin{array}{l}
e_{\theta}=\sqrt{\frac{1}{t_{1}-t_{0}} \int_{t_{0}}^{t_{1}}\left|\theta-\theta_{d}\right|^{2} d \tau} \\
e_{V}=\sqrt{\frac{1}{t_{1}-t_{0}} \int_{t_{0}}^{t_{1}}\left|V-V_{d}\right|^{2} d \tau}
\end{array}\right.
$$

where $\left[t_{0}, t_{1}\right]$ covers the time frame of the overall simulation, $\theta_{d}$ and $V_{d}$ denote the reference signals of the pitch angle and the velocity, respectively.

TABLE II THE SIMULATION SCENARIOS

\begin{tabular}{ccc}
\hline \hline & Scenario I & Scenario II \\
\hline Factor 1 & $\sqrt{ }$ & $\sqrt{ }$ \\
Factor 2 & $\sqrt{ }$ & $\sqrt{ }$ \\
Factor 3 & & $\sqrt{ }$ \\
Comparison & $\sqrt{ }$ \\
\hline \hline
\end{tabular}

Two simulation scenarios are conducted, as indicated in Table II. In Scenario I, three FTAC schemes have been selected in the revised simulation studies. They are: 1) FLS based FTAC with predefined fuzzy rules; 2) self-learning based FTAC with weights being updated; and 3) SCFNN based FTAC with both weights and regressors being adapted. In Scenario II, measurement noises are involved, further examining the performance of the developed SCFNN based FTAC.

\section{B. Simulation Results of Scenario I and Evaluation}

The results demonstrate the use of the designed algorithm in conditions where the aircraft is experiencing the model mismatch and actuator faults. It is shown in Fig. 3 that the three FTAC systems can ensure the safety of the aircraft in the event of actuator faults and model uncertainties. The closed-loop behavior remains satisfactory although the tracking error exhibits a slightly worse transient behavior. As can be seen from Fig. 3, the SCFNN based FTAC outperforms the comparing FTAC schemes, since both weights and regressors can be appropriately adapted in the developed scheme. Fig. 4 highlights how the actuators are managed during the course of fault accommodation. The configured actuators are governed more promptly by the proposed system (the SCFNN based FTAC) in comparison of the other two FTAC schemes.

Moreover, the quantitative performance metrics are listed in Table III. The defined indices achieved by the FLS based FTAC, 
the self-learning based FTAC, and the SCFNN based FTAC are $0.387,0.435$, and 0.144 , respectively. The enhanced rate from the FLS based FTAC to the SCFNN based FTAC is $62.79 \%$ (from 0.387 to 0.144 ), while the improved percentage from the self-learning FTAC to the SCFNN based FTAC is $66.90 \%$ (from 0.435 to 0.144 ). Focusing on the indices of overshoot and settling time, the SCFNN based FTAC can achieve superior performance over that of the comparing FTAC schemes. The quantitative analysis confirms that the presented scheme with adaptation of weights and regressor vectors can not only ensure the safety of the post-fault aircraft, but also preserve a satisfactory degree of tracking performance.

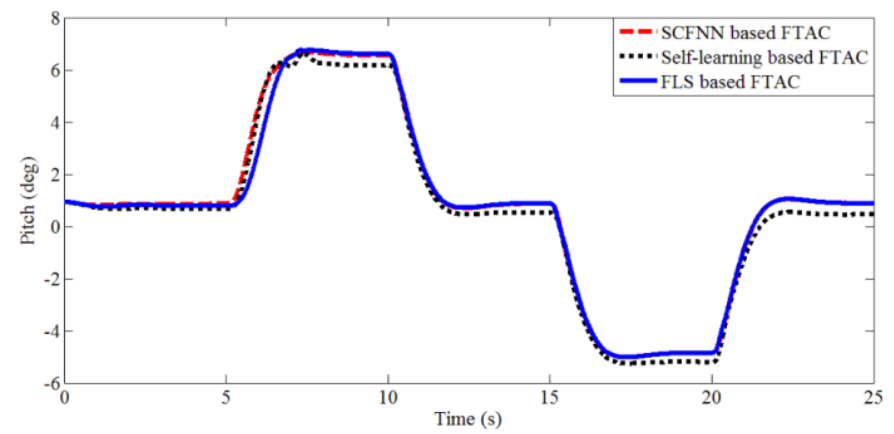

Fig. 3. The profiles of the pitch angle in Scenario I.

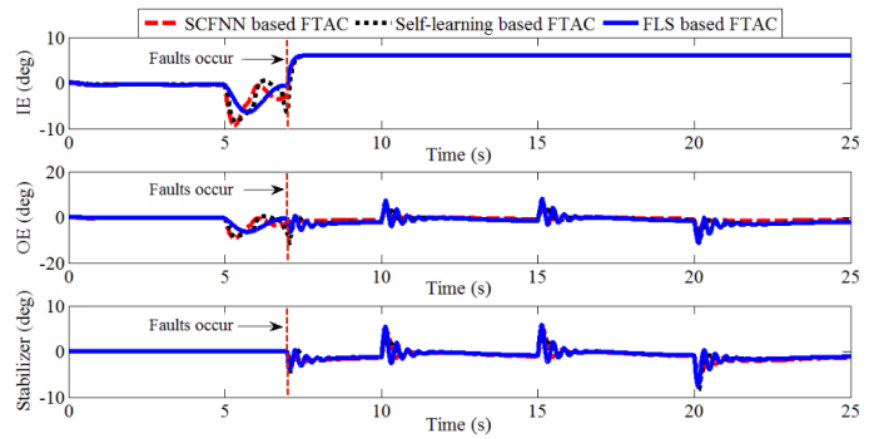

Fig. 4. The responses of the actuators in Scenario I.

The fuzzy rules number $N$ is displayed in Fig. 5. Both the SCFNN based FTAC and self-learning based FTAC can adjust the fuzzy rules. By contrast, the FLS based FTAC is running without any fuzzy rules adjustment. The interesting finding from Fig. 5 is that the SCFNN based FTAC possessing compact fuzzy rules can respond effectively to the faults. As a result, the satisfactory performance of fault accommodation can be achieved with the aid of updating weights and regressor vectors. It is interesting to see from Fig. 6 that the estimated values of $\rho_{1}$ and $\rho_{2}$ respond appropriately by adopting the adaptation mechanism after occurrence of the actuator faults. Figs. 7-10 show that the parameter boundedness of $\bar{c}_{i}, \underline{c}_{i}, \bar{\sigma}_{i}$, and $\underline{\sigma}_{i}$ can be guaranteed.

TABLE III

THE PERFoRMANCE INDICES

\begin{tabular}{cccc}
\hline \hline & Settling time & Overshoot & $e_{\theta}$ \\
\hline $\begin{array}{c}\text { SCFNN based FTAC } \\
\text { Self-learning based } \\
\text { FTAC }\end{array}$ & $1.80 \mathrm{~s}$ & 0 & 0.144 \\
FLS based FTAC & $2.32 \mathrm{~s}$ & 0 & 0.435 \\
\hline \hline
\end{tabular}

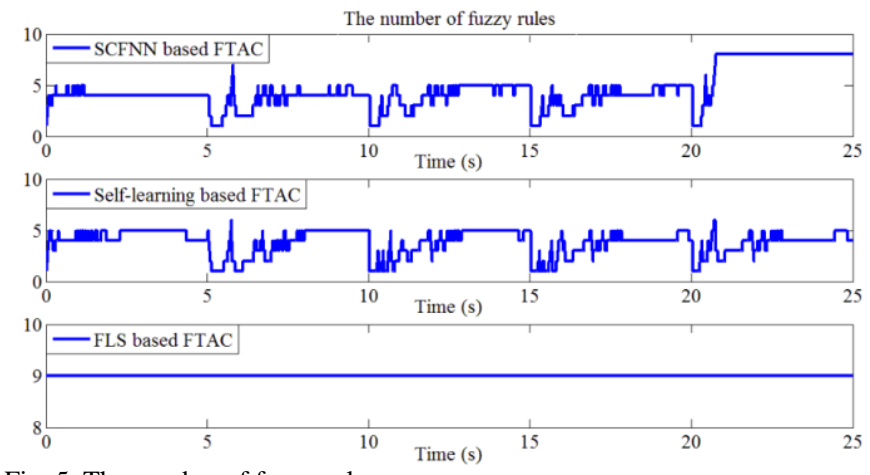

Fig. 5. The number of fuzzy rules.

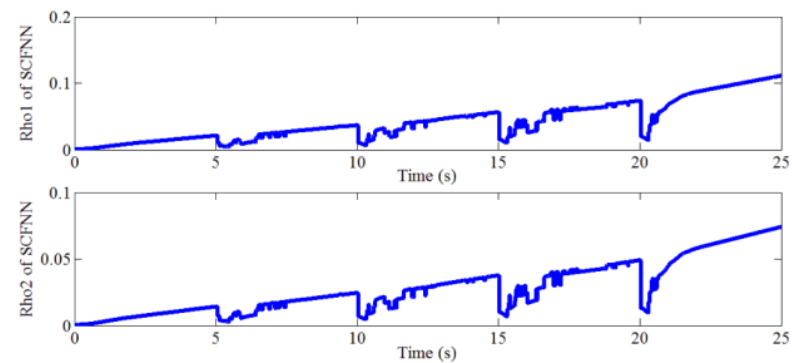

Fig. 6 . The values of $\hat{\rho}_{1}$ and $\hat{\rho}_{2}$ obtained by SCFNNs.
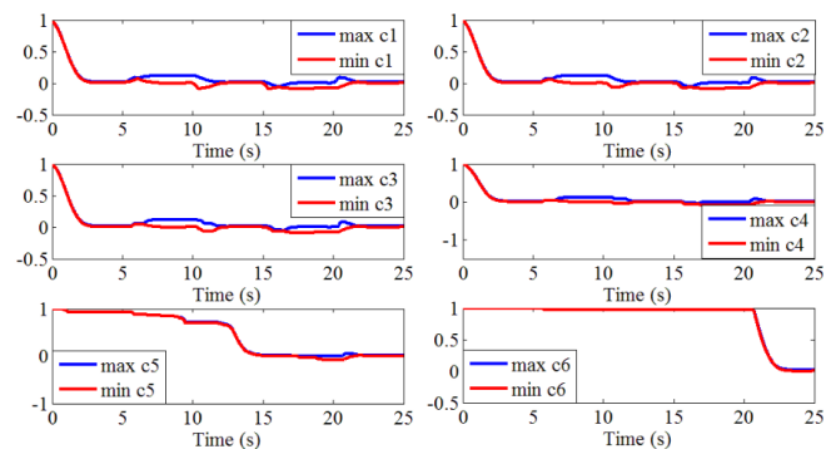

Fig. 7. Center bounds $\bar{c}_{i}$ and $\underline{c_{i}}$ of the first SCFNN.
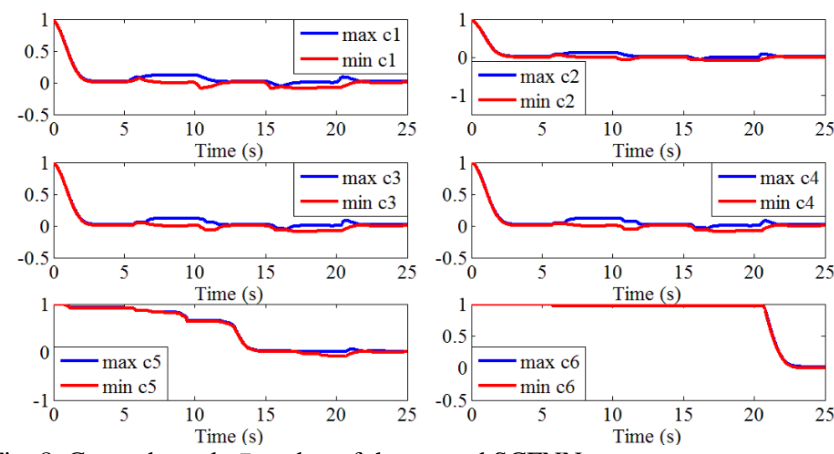

Fig. 8. Center bounds $\bar{c}_{i}$ and $\underline{c_{i}}$ of the second SCFNN.
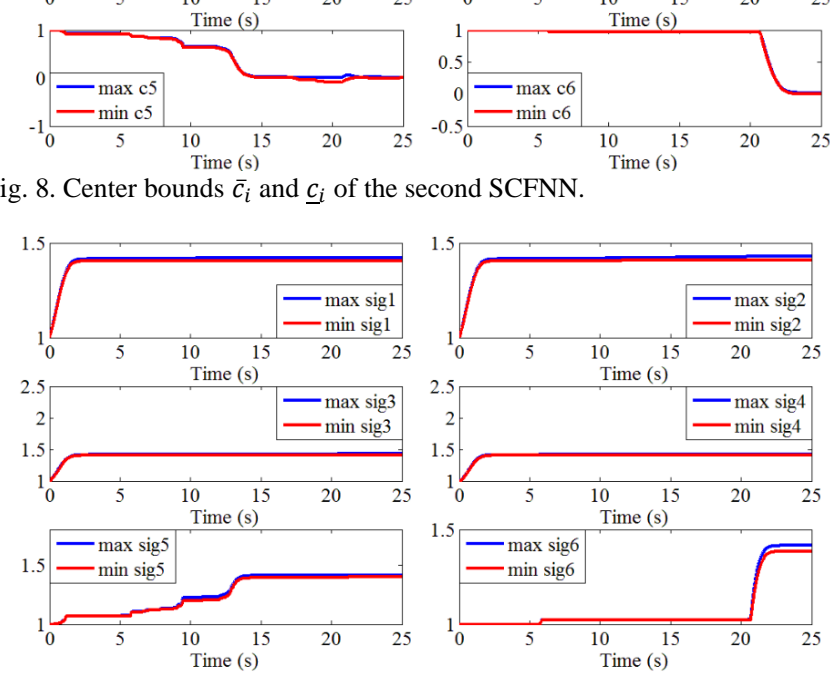

Fig. 9. Width bounds $\bar{\sigma}_{i}$ and $\sigma_{i}$ of the first SCFNN. 

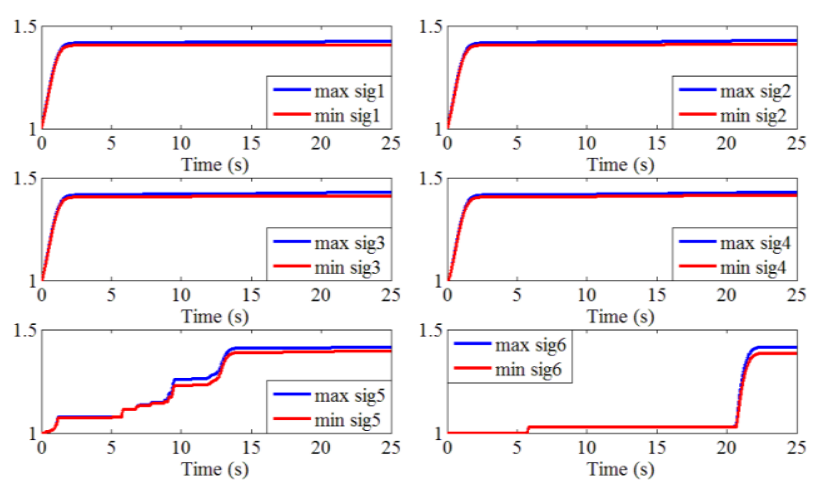

Fig. 10. Width bounds $\bar{\sigma}_{i}$ and $\sigma_{i}$ of the second SCFNN.

\section{Simulation Results of Scenario II and Evaluation}

The robustness of the designed FTAC scheme on sensor noises is examined. Based on Fig. 11, the presented SCFNNFTAC can still preserve an acceptable level of tracking performance when model variation, actuator malfunctions, and noisy measurements are involved simultaneously. It is depicted in Fig. 12 that the peak value of the tracking error of pitch angle is nearly $0.26^{\circ}$. Since the noisy measurements are used for feedback, the tracking error can only converge to a small vicinity of zero. On the other hand, graceful performance degradation is an option in FTAC design without inducing overload to fault-free actuators. As can be observed from Fig. 13 , the actuators, which are affected by unfavorable conditions of faults and sensor noises, can still be governed to counteract malfunctions. The values of of $\hat{\rho}_{1}$ and $\hat{\rho}_{2}$ are displayed in Fig. 14. It is shown that $\hat{\rho}_{1}$ and $\hat{\rho}_{2}$ can be adapted in response to the unfavorable situation of Scenario II, such that the FTAC law can react correspondingly.

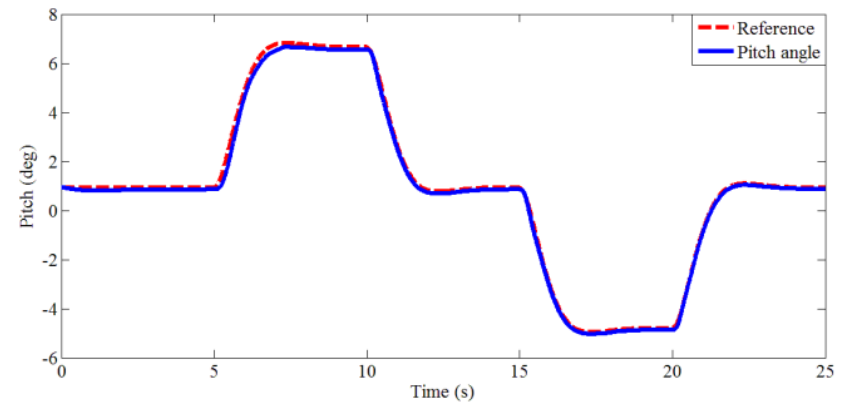

Fig. 11. The response of the pitch angle in Scenario II.

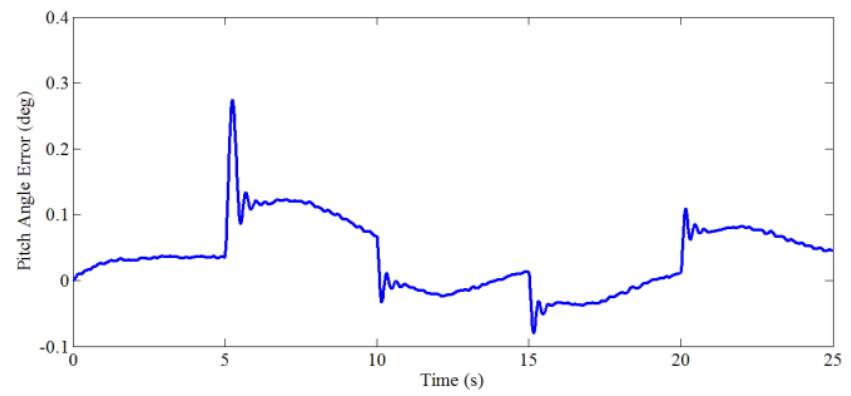

Fig. 12. Tracking error of pitch angle in Scenario II.

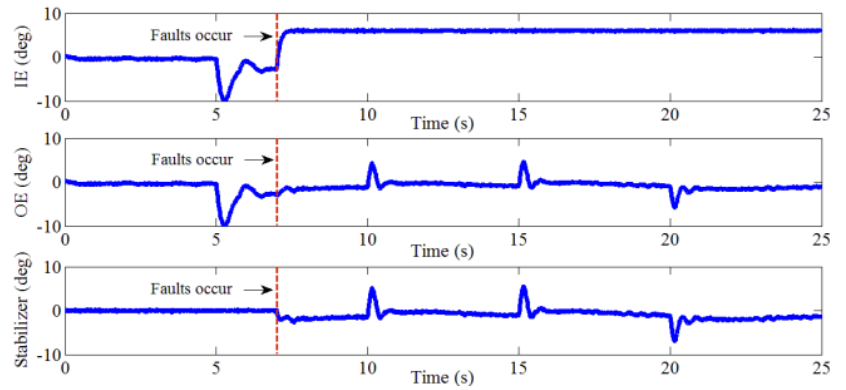

Fig. 13. Actuators deflections in Scenario II.
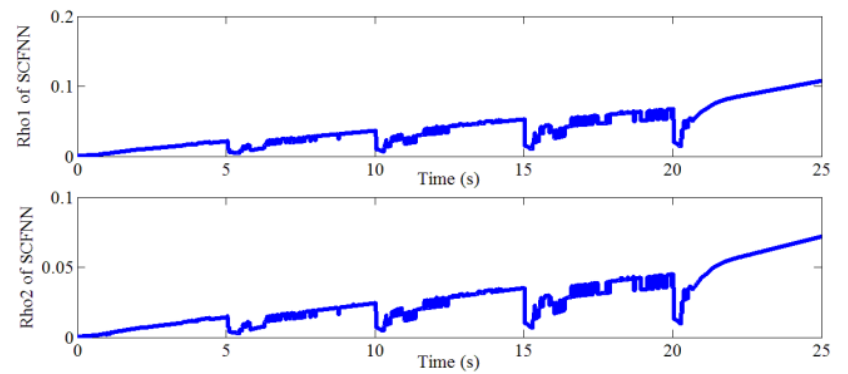

Fig. 14. The values of $\hat{\rho}_{1}$ and $\hat{\rho}_{2}$ in Scenario II.

\section{CONCLUSIONS AND FUture WORKS}

Actuator malfunctions and aircraft model uncertainties have a detrimental impact on aircraft flight safety. To better address these difficulties, self-constructing fuzzy neural networks (SCFNNs) and multivariable sliding mode control (SMC) with adaptive techniques are exploited to enable integration into a fault-tolerant aircraft control (FTAC) system. The presented strategy has three benefits. 1) By introducing the upper bounds of the unknown function norms, the SCFNNs assisted by adaptive algorithms are exploited to approximate the information of actuator faults and model uncertainties; 2) The finite-time stability of the faulty aircraft can be ensured and an acceptable level of tracking performance can be preserved; and 3) The SMC method is presented under multivariable situations, instead of the decoupled single-input and single-output method. Numerical simulations show that the proposed scheme is able to deal with scenarios involving actuator faults and model uncertainties.

Although the presented scheme is capable of tolerating flight actuator faults, issues of sensor fault accommodation have not yet been addressed in the design. Investigation of these factors which may affect the performance of the FTAC system is one of our future works. In addition, experimental testbed development and experimental validation of the proposed algorithm are also our current and future research works.

\section{ACKNOWLEDGMENTS}

The authors would like to express their sincere gratitude to the Associate Editor and the anonymous reviewers whose insightful comments have helped to improve the quality of this paper considerably. The authors would like to thank Sean Zhang for the proofreading that helped to improve the presentation of this paper significantly. 


\section{REFERENCES}

[1] J. Jiang and X. Yu, "Fault-Tolerant Control Systems: A Comparative Study Between Active and Passive Approaches," Annu. Rev. Control, vol. 36, no. 1, pp. 60-72, Apr. 2012.

[2] X. Yu and J. Jiang, "A Survey of Fault-Tolerant Controllers Based on Safety-Related Issues," Annu. Rev. Control, vol. 39, no. 1, pp. 46-57, Apr. 2015.

[3] Y. M. Zhang and J. Jiang, "Bibliographical Review on Reconfigurable Fault-Tolerant Control Systems," Апnи. Rev. Control, vol. 32, no. 2, pp. 229-252, Dec. 2008.

[4] J. Jiang and Q. Zhao, "Design of Reliable Control Systems Possessing Actuator Redundancy," J. Guid. Control Dyn., vol. 23, no. 4, pp. 709-718, Jul. 2000.

[5] F. Liao, J. L. Wang, and G. H. Yang, "Reliable Robust Flight Tracking Control: An LMI Approach," IEEE Trans. Control Syst. Technol., vol. 10, no. 1, pp. 76-89, Jan. 2002.

[6] X. Yu and Y. M. Zhang, "Design of Passive Fault-Tolerant Flight Controller Against Actuator Failures," Chinese J. Aeronaut., vol. 28, no. 1, pp. 180-190, Feb. 2015.

[7] F. A. D. Almeida and D. Leibling, "Fault-Tolerant Model Predictive Control with Flight-Test Results," J. Guid. Control Dyn., vol. 33, no. 2, pp. 363-375, Mar. 2010.

[8] L. G. Sun, C. C. d. Visser, Q. P. Chu, and W. Falkena, "Hybrid SensorBased Backstepping Control Approach with its Application to FaultTolerant Flight Control," J. Guid. Control Dyn., vol. 37, no. 1, pp. 59-71, Jan. 2014.

[9] L. G. Sun, C. C. d. Visser, Q. P. Chu, and J. A. Mulder, "Joint Sensor Based Backstepping for Fault-Tolerant Flight Control," J. Guid. Control Dyn., vol. 38, no. 1, pp. 62-75, Jan. 2015.

[10] D. Shore and M. Bodson, "Flight Testing of a Reconfigurable Control System on an Unmanned Aircraft," J. Guid. Control Dyn., vol. 28, no. 4, pp. 698-707, Jul. 2005.

[11] G. Tao, S. H. Chen, and S. M. Joshi, "An Adaptive Control Scheme for Systems with Unknown Actuator Failures," Automatica, vol. 38, no. 6, pp. 1027-1034, June 2002.

[12] G. Tao, S. M. Joshi, and X. Ma, "Adaptive State Feedback and Tracking Control of Systems with Actuator Failures," IEEE Trans. Autom. Control, vol. 46, no. 1, pp. 78-95, Jan. 2001.

[13] X. Yu, Y. M. Zhang, and Z. X. Liu, "Fault-Tolerant Flight Control Design with Explicit Consideration of Reconfiguration Transients," J. Guid. Control Dyn., vol. 39, no. 3, pp. 556-563, Mar. 2016.

[14] X. Yu, Z. X. Liu, and Y. M. Zhang, "Fault-Tolerant Flight Control with Finite-Time Adaptation under Actuator Stuck Failures," IEEE Trans. Control Syst. Technol., vol. 25, no. 4, pp. 1431-1440, Jul. 2017.

[15] H. Alwi and C. Edwards, "Fault Detection and Fault-Tolerant Control of a Civil Aircraft Using a Sliding-Mode-Based Scheme," IEEE Trans. Control Syst. Technol., vol. 16, no. 3, pp. 499-510, May 2008.

[16] M. T. Hamayun, C. Edwards, and H. Alwi, "Augmentation Scheme for Fault-Tolerant Control Using Integral Sliding Modes," IEEE Trans. Control Syst. Technol., vol. 22, no. 1, pp. 307-313, Jan. 2014.

[17] X. Yu, Y. Fu, and Y. M. Zhang, "Aircraft Fault Accommodation with Consideration of Actuator Control Authority and Gyro Availability," IEEE Trans. Control Syst. Technol., Online Published, DOI: 10.1109/TCST.2017.2707378, 2017.

[18] X. Yu and J. Jiang, "Fault-Tolerant Flight Control System Design Against Control Surface Impairments," IEEE Trans. Aerosp. Electron. Syst., vol. 48, no. 2, pp. 1031-1051, Apr. 2012.

[19] H. Badihi, Y. M. Zhang, and H. Hong, "Wind Turbine Fault Diagnosis and Fault-Tolerant Torque Load Control Against Actuator Faults," IEEE Trans. Control Syst. Technol., vol. 23, no. 4, pp. 1351-1372, Jul. 2015.

[20] H. Badihi, Y. M. Zhang, and H. Hong, "Fuzzy Gain-Scheduled Active Fault-Tolerant Control of a Wind Turbine," J. Franklin Inst., vol. 351, no. 7, pp. 3677-3706, Jul. 2014.

[21] W. A. Kwong, K. M. Passino, E. G. Laukonen, and S. Yurkovich, "Expert Supervision of Fuzzy Learning Systems for Fault Tolerant Aircraft Control," Proc. IEEE, vol. 83, no. 3, pp. 466-483, Mar. 1995.

[22] H. J. Rong, N. Sundararajan, P. Saratchandran, and G. B. Huang, "Adaptive Fuzzy Fault-Tolerant Controller for Aircraft Autolanding under Failures," IEEE Trans. Aerosp. Electron. Syst., vol. 43, no. 4, pp. 1586-1603, Oct. 2007.

[23] Q. K. Shen, B. Jiang, and V. Cocquempot, "Fuzzy Logic System-Based Adaptive Fault-Tolerant Control for Near-Space Vehicle Attitude Dynamics with Actuator Faults," IEEE Trans. Fuzzy Syst., vol. 21, no. 2, pp. 289-300, Apr. 2013.
[24] Q. K. Shen, B. Jiang, and V. Cocquempot, "Fault-Tolerant Control for TS Fuzzy Systems with Application to Near-Space Hypersonic Vehicle with Actuator Faults," IEEE Trans. Fuzzy Syst., vol. 20, no. 4, pp. 652665, Aug. 2012.

[25] Q. K. Shen, B. Jiang, and P. Shi, "Adaptive Fault Diagnosis for T-S Fuzzy Systems with Sensor Faults and System Performance Analysis," IEEE Trans. Fuzzy Syst., vol. 22, no. 2, pp. 274-285, Apr. 2014.

[26] Q. K. Shen, B. Jiang, P. Shi, and J. Zhao, "Cooperative Adaptive Fuzzy Tracking Control for Networked Unknown Nonlinear Multiagent Systems with Time-Varying Actuator Faults," IEEE Trans. Fuzzy Syst., vol. 22, no. 3, pp. 494-504, June 2013.

[27] S. Zeghlache, K. Kara, and D. Saigaa, "Fault Tolerant Control Based on Interval Type-2 Fuzzy Sliding Mode Controller for Coaxial Trirotor Aircraft," ISA Trans., vol. 51, no. 11, pp. 215-231, Nov. 2015.

[28] L. Y. Hao, J. H. Park, and D. Ye, "Fuzzy Logic Systems-Based Integral Sliding Mode Fault-Tolerant Control for a Class of Uncertain Nonlinear Systems," IET Control Theory Appl., vol. 10, no. 3, pp. 300-311, Mar. 2016.

[29] S. Barghandan, M. A. Badamchizadeh, and M. R. Jahed-Motlagh, "Improved Adaptive Fuzzy Sliding Mode Controller for Robust Fault Tolerant of a Quadrotor," Int. J. Control, Autom. Syst., vol. 15, no. 1, pp. 427-441, Feb. 2017.

[30] C. M. Lin and C. F. Hsu, "Self-Learning Fuzzy Sliding-Mode Control for Antilock Braking Systems," IEEE Trans. Control Syst. Technol., vol. 11, no. 2, pp. 273-281, Mar. 2003.

[31] C. S. Chen, "Robust Self-Organizing Neural-Fuzzy Control with Uncertainty Observer for MIMO Nonlinear Systems," IEEE Trans. Fuzzy Syst., vol. 19, no. 4, pp. 694-706, Aug. 2011.

[32] Y. M. Zhang and X. R. Li, "A Fast U-D Factorization-Based Learning Algorithm with Applications to Nonlinear System Modeling and Identification," IEEE Trans. Neural Netw., vol. 10, no. 4, pp. 930-938, Jul. 1999.

[33] N. Wang, M. J. Er, J. C. Sun, and Y. C. Liu, "Adaptive Robust Online Constructive Fuzzy Control of a Complex Surface Vehicle System," IEEE Trans. Cybern., vol. 46, no. 7, pp. 1511-1523, Jul. 2016.

[34] N. Wang and M. J. Er, "Self-Constructing Adaptive Robust Fuzzy Neural Tracking Control of Surface Vehicles with Uncertainties and Unknown Disturbances," IEEE Trans. Control Syst. Technol., vol. 23, no. 3, pp. 991-1002, May 2015.

[35] I. Szaszi, A. Marcos, G. J. Balas, and J. Bokor, "Linear ParameterVarying Detection Filter Design for a Boeing 747-100/200 Aircraft," J. Guid. Control Dyn., vol. 28, no. 3, pp. 461-470, May 2005.

[36] J. Y. Shin, C. Belcastro, and T. Khong, "Closed-Loop Evaluation of an Integrated Failure Identification and Fault Tolerant Control System for a Transport Aircraft," in Proc. AIAA Guid. Nav. Control Conf., Keystone, US, Aug. 2006, pp. AIAA Paper 2006-6310.

[37] X. Yu and J. Jiang, "Hybrid Fault-Tolerant Flight Control System Design Against Partial Actuator Failures," IEEE Trans. Control Syst. Technol., vol. 20, no. 4, pp. 871-886, Jul. 2012.

[38] C. F. Hsu, "Self-Organizing Adaptive Fuzzy Neural Control for a Class of Nonlinear Systems," IEEE Trans. Neural Netw., vol. 18, no. 4, pp. 1232-1241, Jul. 2007.

[39] A. G. Barto, R. S. Sutton, and C. W. Anderson, "Neuronlike Adaptive Elements that Can Solve Difficult Learning Control Problems," IEEE Trans. Syst. Man, Cybern., vol. 13, no. 5, pp. 834-846, Sept./Oct. 1983.

[40] C. C. Lee, "Fuzzy Logic in Control Systems: Fuzzy Logic Controller Part I," IEEE Trans. Syst., Man, Cybern., vol. 20, no. 2, pp. 404-418, Mar./Apr. 1990.

[41] S. G. Khan, G. Herrmann, F. L. Lewis, T. Pipe, and C. Melhuish, "Reinforcement Learning and Optimal Adaptive Control: An Overview and Implementation Examples," Annu. Rev. Control, vol. 36, no. 1, pp. 42-59, Apr. 2012.

[42] Y. Pan, M. J. Er, D. Huang, and Q. Wang, "Adaptive Fuzzy Control with Guaranteed Convergence of Optimal Approximation Error," IEEE Trans. Fuzzy Syst., vol. 19, no. 5, pp. 807-818, Oct. 2011.

[43] C. C. Cheng and S. H. Chien, "Adaptive Sliding Mode Controller Design Based on T-S Fuzzy System Models," Automatica, vol. 42, no. 6, pp. 1005-1010, June 2006.

[44] I. Nagesh and C. Edwards, "A Multivariable Super-Twisting Sliding Mode Approach," Automatica, vol. 50, no. 3, pp. 984-988, Mar. 2014. 